\title{
WestVirginiaUniversity
}

THE RESEARCH REPOSITORY @ WVU

Graduate Theses, Dissertations, and Problem Reports

2010

\section{Power simulator upgrade for smart grid algorithm development and testing}

Michael Joseph Spencer

West Virginia University

Follow this and additional works at: https://researchrepository.wvu.edu/etd

\section{Recommended Citation}

Spencer, Michael Joseph, "Power simulator upgrade for smart grid algorithm development and testing" (2010). Graduate Theses, Dissertations, and Problem Reports. 4659.

https://researchrepository.wvu.edu/etd/4659

This Thesis is protected by copyright and/or related rights. It has been brought to you by the The Research Repository @ WVU with permission from the rights-holder(s). You are free to use this Thesis in any way that is permitted by the copyright and related rights legislation that applies to your use. For other uses you must obtain permission from the rights-holder(s) directly, unless additional rights are indicated by a Creative Commons license in the record and/ or on the work itself. This Thesis has been accepted for inclusion in WVU Graduate Theses, Dissertations, and Problem Reports collection by an authorized administrator of The Research Repository @ WVU. For more information, please contact researchrepository@mail.wvu.edu. 


\title{
Power Simulator Upgrade for Smart Grid Algorithm Development and Testing
}

\author{
Michael Joseph Spencer \\ Thesis submitted to the \\ College of Engineering and Mineral Resources \\ at West Virginia University \\ in partial fulfillment of the requirements \\ for the degree of \\ Master of Science \\ in \\ Electrical Engineering \\ Muhammad A. Choudhry, Ph.D. \\ Daryl S. Reynolds, Ph.D. \\ Ali Feliachi, Ph.D., Chair
}

Lane Department of Computer Science and Electrical Engineering

\author{
Morgantown, West Virginia \\ 2010
}

Keywords: smart grid, power system, hardware prototype, real-time control, reconfigurable power system, agent communication, controller area network, power conversion, power electronics.

Copyright 2010 Michael Joseph Spencer 


\begin{abstract}
Power Simulator Upgrade for Smart Grid Algorithm Development and Testing

Michael Joseph Spencer
\end{abstract}

This thesis describes the conversion of WVU's analog power simulator into a micro-grid of the future test bed by installing digital relays and intelligent electronic switches. The simulator is a hardware representation of the grid which contains traditional hardware, both digital and analog as well as the recent addition of highly connected, via Ethernet and potentially wireless communication, smart switching and monitoring devices. These new devices were chosen specifically for their cyber security capability to explore that facet of smart grid development. It is important to note that this simulator is a hardware implementation and as such is capable of testing smart grid ideas in the most realistic setting available without affecting real customers. This simulator also has the potential to have renewable resources like wind and solar as well as fuel cell and battery storage distributed resources tied in to test smart grid adaptability to these next generation ideas.

New digital relays were installed. Micro controller units and energy meter integrated circuits were investigated based on the desire to provide many modes of communication and as much processing power as was available in a small package. Solid state switches were designed and implemented for speed, compactness and reduced power consumption. 


\section{Dedication}

This thesis is dedicated to my parents Terry Michael Spencer and Anna May Spencer. 


\section{Acknowledgment}

I would like to thank my advisor, Dr. Ali Feliachi, for allowing me to pursue this project and for his help, encouragement, understanding and support through all of the obstacles encountered during this time. I would like to thank Dr. Muhammad A. Choudhry for his continued guidance and valuable suggestions. I would like to thank Dr. Daryl S. Reynolds for his encouragement and ideas on communication that I've tried to incorporate into this project. I would like to express my thanks to all of faculty members and students at APERC for their help and support, particularly Prof. Ed Sneckenberger for his endless enthusiasm and encouragement. I would also like to thank Dr. Powsiri Klinkhachorn and Dr. Roy S. Nutter, Jr. for their free and open advice anytime I would show up at their door. Finally I'd like to thank Emily D. Pertl, PhD for her help getting this document into a readable format.

All that said I am most thankful to my parents for their love, encouragement and support. 


\section{Table of Contents}

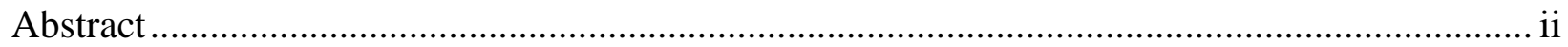

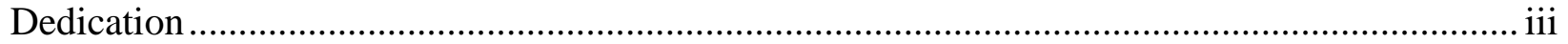

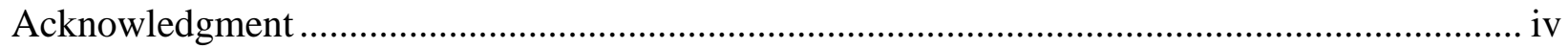

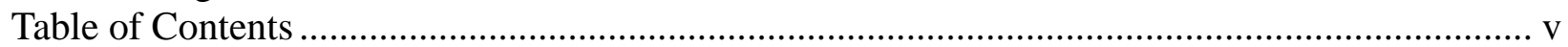

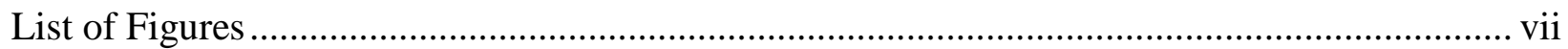

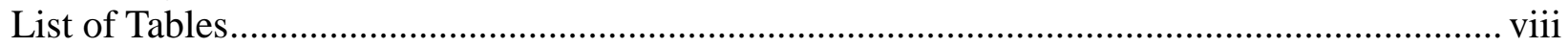

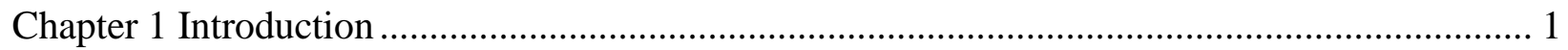

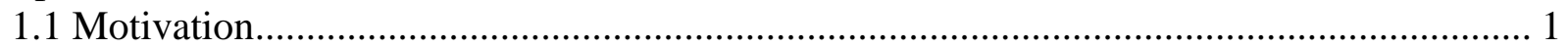

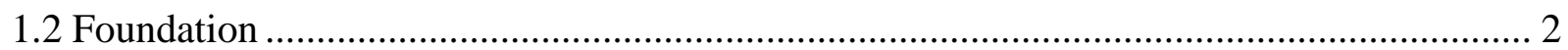

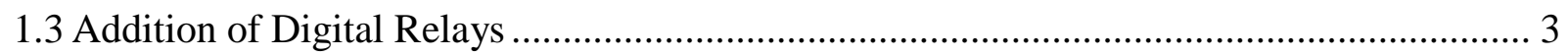

1.4 Addition of Intelligent Electronic Switches..................................................................... 4

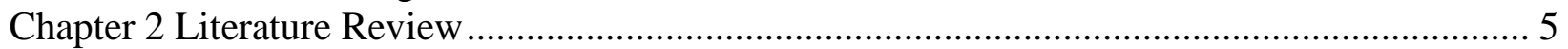

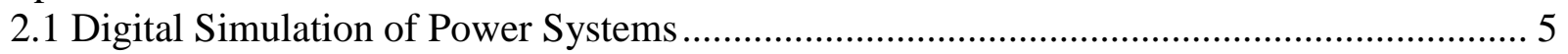

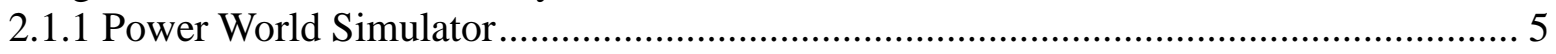

2.1.2 PAT Power Analysis Toolbox........................................................................ 5

2.1.3 RTDS Real Time Digital Simulator ………………..................................................... 6

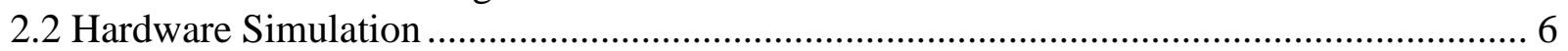

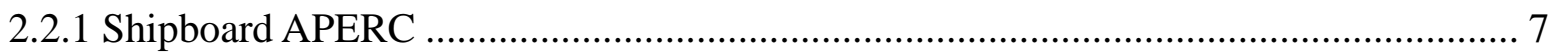

2.2.2 Hampden Engineering Corporation Power System Simulator ........................................ 7

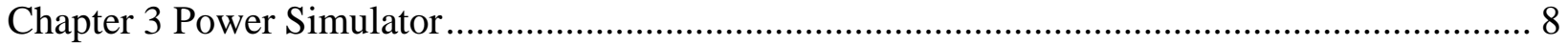

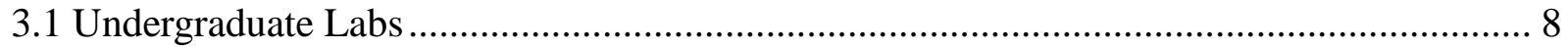

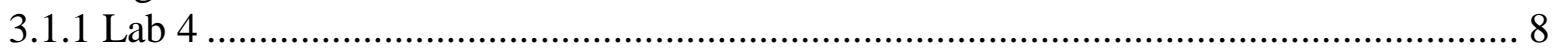

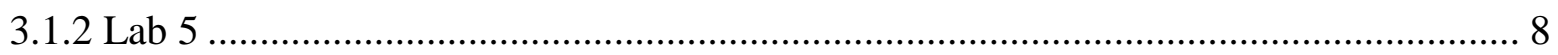

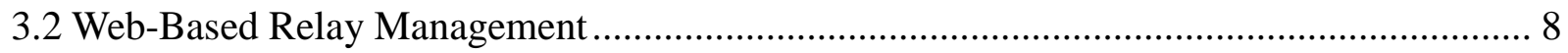

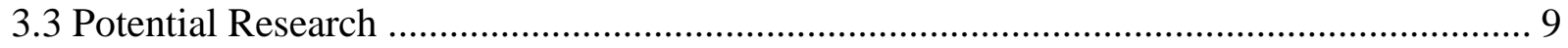

3.3.1 Real time testing of MAS and other control algorithms .............................................. 9

3.3.1.1 Reconfiguration ................................................................................................. 9

3.3.1.2 Optimum Power Flow, Load Shedding and Distributed Generation ...................... 9

3.3.1.3 Communication Experimentation ................................................................... 10

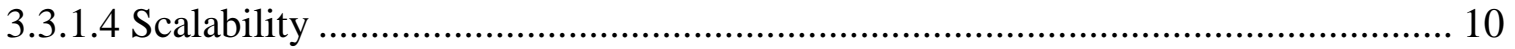

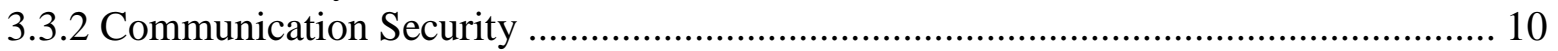

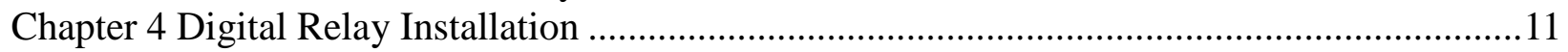

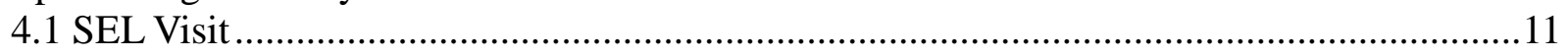

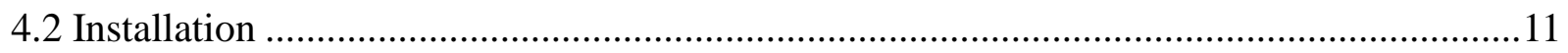

Chapter 5 Intelligent Electronic Switch Hardware Selection ...................................................... 20

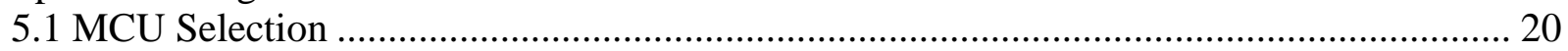

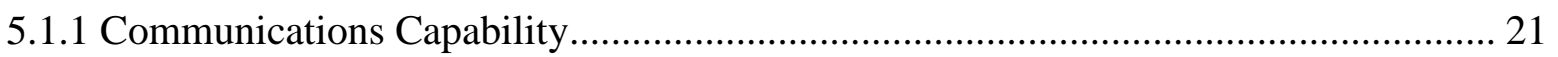

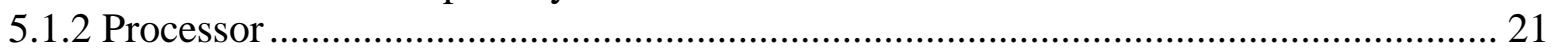

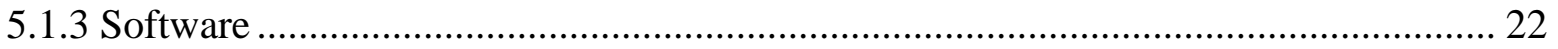

5.2 Triac and opto-isololator selection............................................................................ 22

5.3 Metering Integrated Circuit Discovery and Selection ................................................... 22

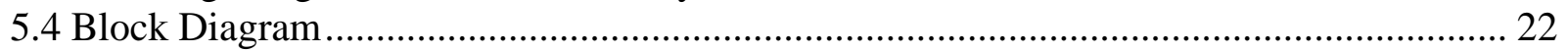

Chapter 6 Circuit Design and Board Fabrication.................................................................... 24

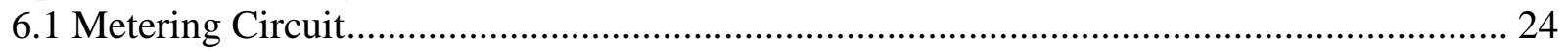




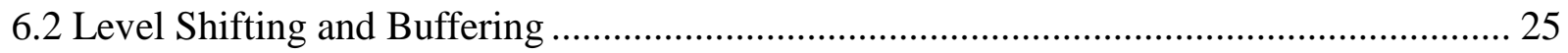

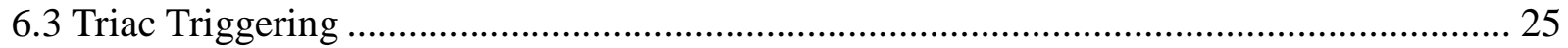

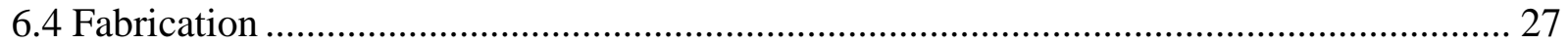

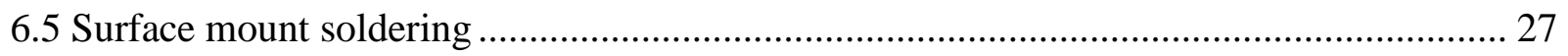

6.6 Reworking Surface Mount Parts............................................................................... 34

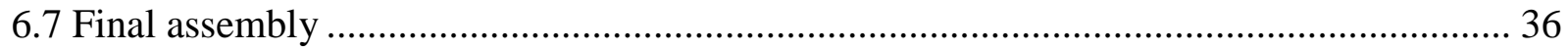

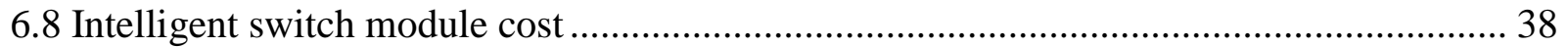

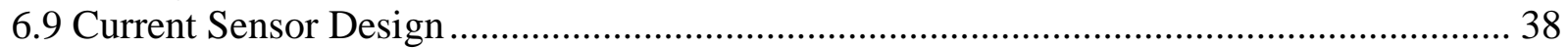

6.10 Clock and Distribution Circuit Design ....................................................................... 39

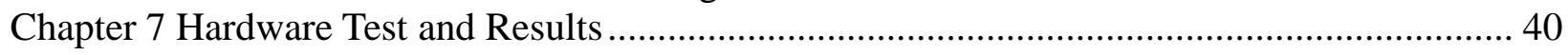

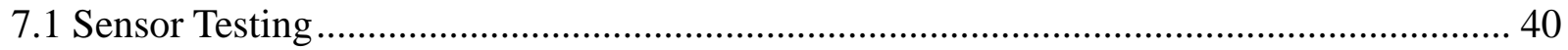

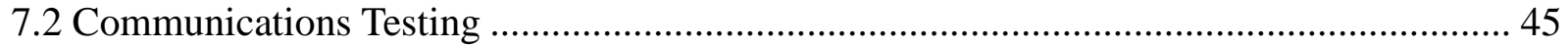

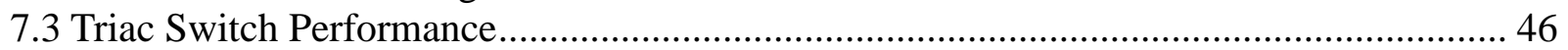

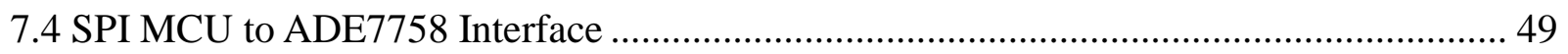

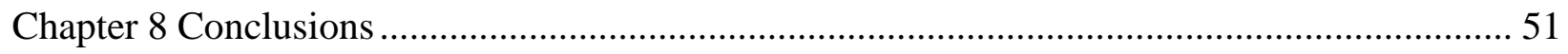

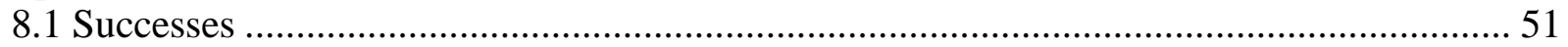

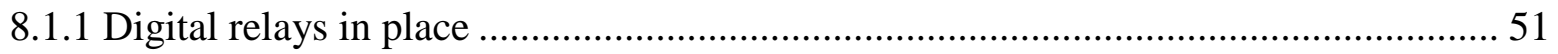

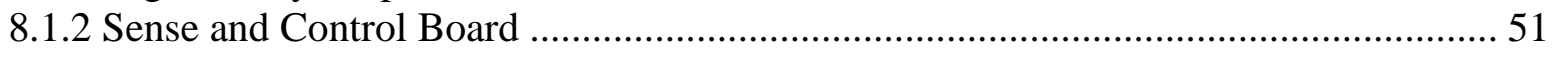

8.1.3 Designed fabricated and tested current sensors ....................................................... 51

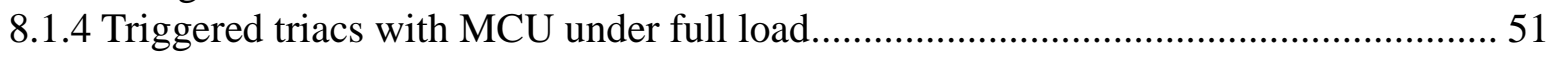

8.1.5 Modified code for serial to Ethernet operation ......................................................... 52

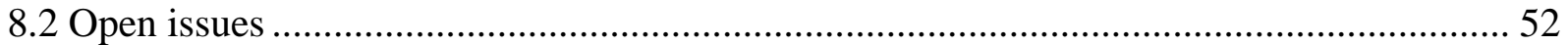

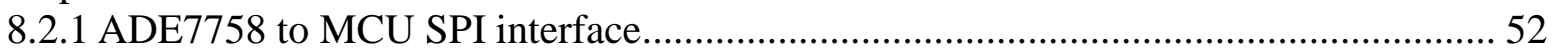

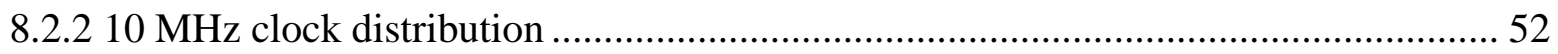

8.3 Future work

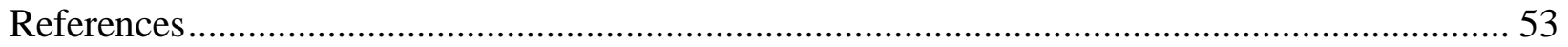




\section{List of Figures}

Figure 1: Shipboard simulator developed by Pradeep Pant (1) .............................................. 2

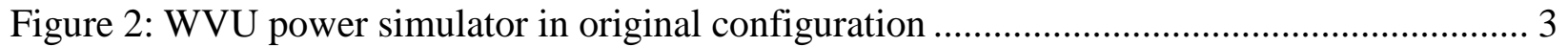

Figure 3: Power simulator with added digital relays ......................................................... 4

Figure 4: A peek behind the curtain at the back of the power simulator front panel .................. 12

Figure 5: SEL 351S the most difficult relay installation ................................................... 13

Figure 6: Panel junction that had to be cut for installation ............................................... 14

Figure 7: Panel junction close-up .............................................................................. 15

Figure 8: Panel junction with new strut bolted in place .................................................. 16

Figure 9: View of extended strut from behind the panel ................................................. 17

Figure 10: View of shortened strut and end panel reinforcement ....................................... 18

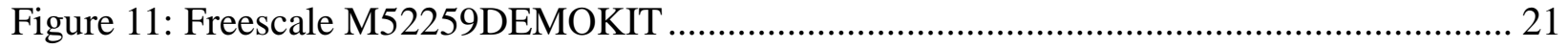

Figure 12: Block diagram of intelligent electronic switch .............................................. 23

Figure 13: Circuit recommended by Analog Devices (2) ..................................................... 25

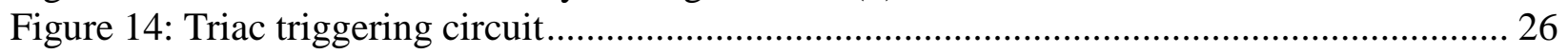

Figure 15: Sense and control printed circuit board layout ............................................... 27

Figure 16: Bare printed circuit board ready for surface mount soldering; the transparent green

coating is the solder mask......................................................................................... 28

Figure 17: Applying solder paste to the solder pad of one of the large capacitors ..................... 29

Figure 18: Placing the capacitor on it's solder pads with solder paste in place .......................... 30

Figure 19: Board in oven with all of the surface mount parts in place ready for solder reflow ... 31

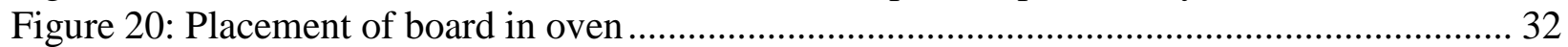

Figure 21: After cooling a satisfactory SMT soldered board, note the two resistor capacitor pairs

that are angled together probably due to just a little too much solder on the right side of each... 33

Figure 22: SMT printed circuit board rework setup ....................................................... 35

Figure 23: SMT printed circuit board rework setup closeup ................................................ 36

Figure 24: The sense and control board with SMT parts, opto-isolators and triacs installed on

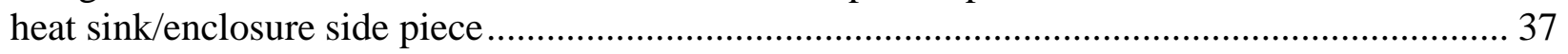

Figure 25: Current sensors being bench tested with the ADE7758 evaluation board ................. 39

Figure 26: Sensor test setup using ADE7758 evaluation board.............................................. 41

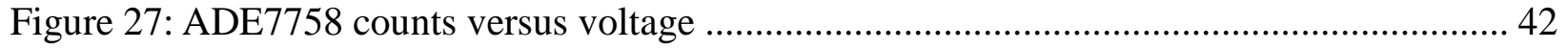

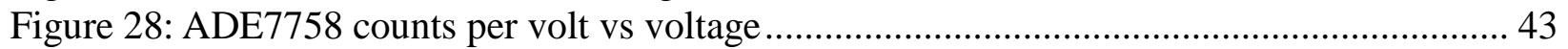

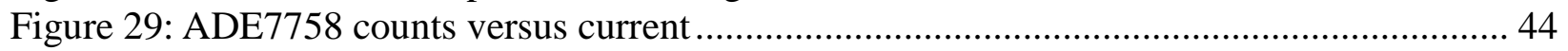

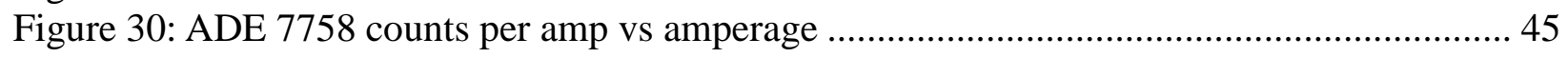

Figure 31: Test setup for Ethernet to serial communications rerouting .................................. 46

Figure 32: Three phase triac turn on waveforms with resistive load ..................................... 47

Figure 33: Three phase triac turn off waveforms with resistive load .................................... 48

Figure 34: Three phase triac turn off with inductive load ................................................... 49

Figure 35: SPI communication interface test setup ......................................................... 50 


\section{List of Tables}

Table 1: List of equipment donated by SEL ..................................................................... 3

Table 2: Original electromechanical relay vs. SEL digital counterpart ...................................11

Table 3: Wiring between the M52259DEMOKIT and the sense and control board................... 37

Table 4: Parts breakdown for one intelligent electronic switch are as follows........................... 38 


\section{Chapter 1 Introduction}

The United States government has recently decided to invest billions of dollars into the existing, and maybe antiquated, power grid in an effort to make it more efficient and reliable. The name given to the new power grid is the Smart Grid, the main idea being that through more intelligent devices and communication both on the consumer and utilities side the grid can be made more reliable with autonomous reconfiguration and disaster mediation and more efficient with potential consumer interaction on a real time basis. This thesis proposes to deliver a platform where ideas on automated reconfiguration and control via multi-agent systems (MAS's) or other control algorithms can be tested. This will be accomplished by converting a small scale analog distribution simulator of 1970's technology into a micro-grid of the future by the addition of modern digital relay equipment and micro-controlled switches.

\subsection{Motivation}

The idea for the conversion came about as a logical next step from the shipboard simulator developed by Pradeep Pant (1). Figure 1 shows the shipboard simulator it was developed to test reconfiguration schemes of shipboard power. It has microprocessor controlled switches to route DC power from the two main busses to the many distributed loads. The switches communicate over a CAN bus to coordinate reconfiguration in case of damage to the system.

Success with the shipboard test bed has encouraged the pursuit of a similar platform that can handle AC distribution systems. An important property of multi-agent systems is the widespread use of communication. By using multiple communication paths that are jointly optimized higher performance can be achieved. There was a real need for a hardware platform to test multi-agent and other algorithms as well as communication schemes on the unique problems posed by a three phase power system. This was the motivation to upgrade the existing analog power simulator that mimics a typical distribution system. 


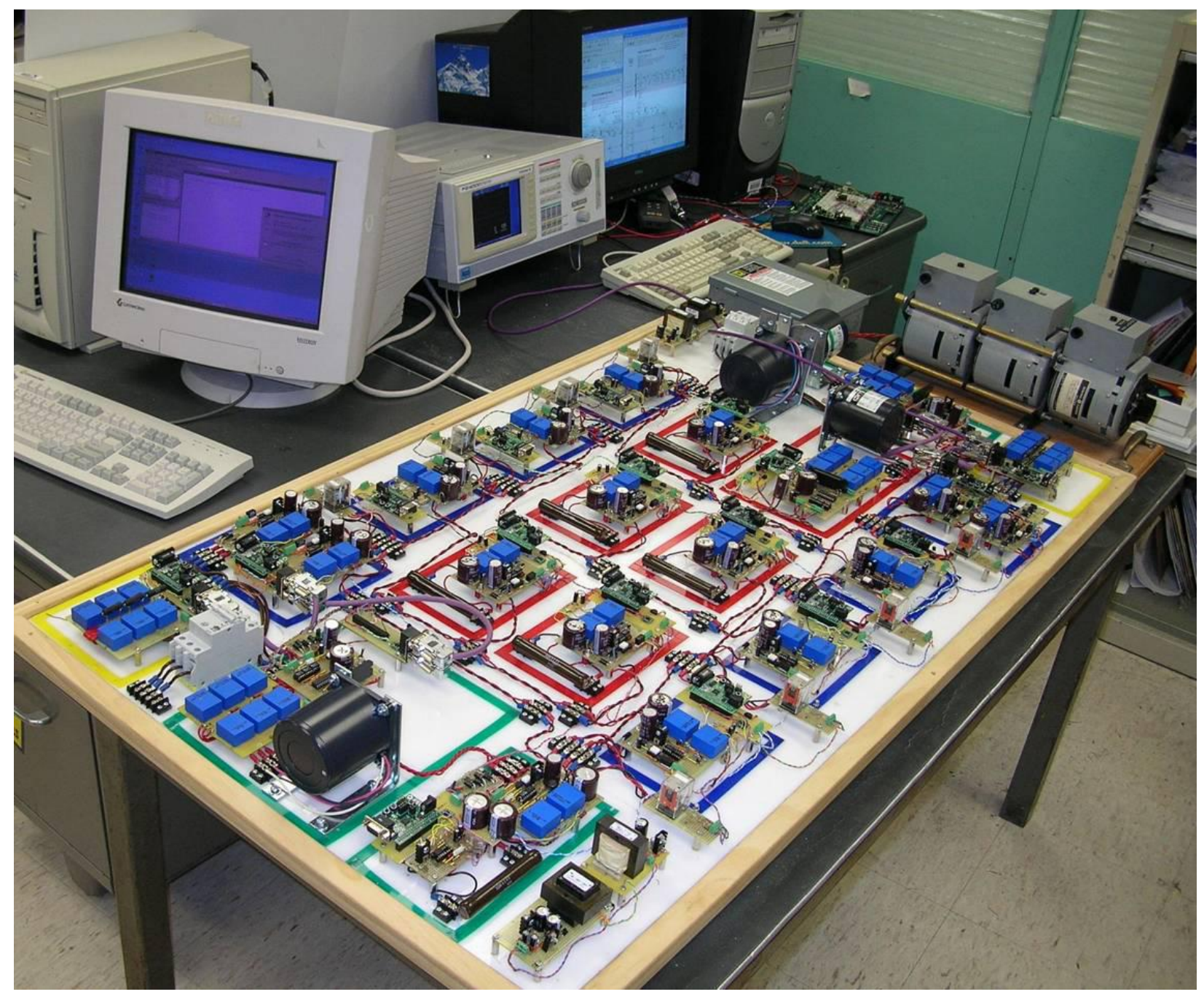

Figure 1: Shipboard simulator developed by Pradeep Pant (1)

\subsection{Foundation}

The Lane Department of Computer Science and Electrical Engineering at West Virginia University maintains an analog power simulator that was donated to the university and installed in the 1970s. The simulator, as shown in Figure 2, is a low power hardware replica of a distribution system that contains commercial, industrial and residential loads. Power can be supplied to the loads from different internal and external circuits and generators and routed in a variety of ways. The simulator is at this time being retrofitted to represent a micro-grid of the future by installing digital relays, intelligent electronic devices, distributed energy resources (generation and storage), and potentially a FACTS device. The new digital hardware will be 
integrated with the older electromechanical hardware as is found in real world power systems. This will allow the system to be used for research into autonomous reconfiguration schemes.

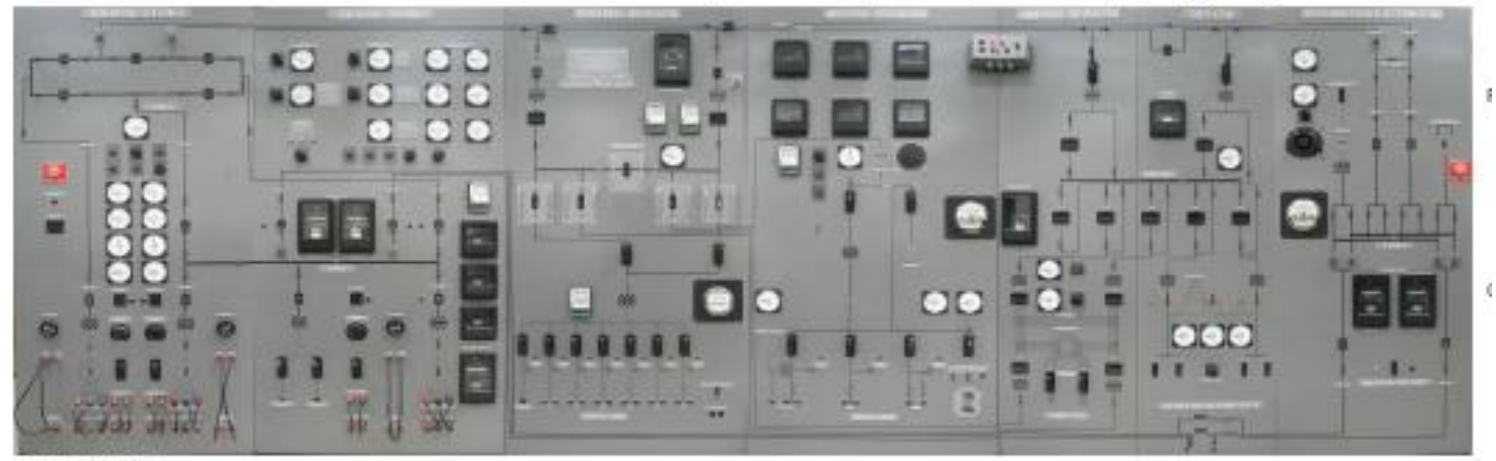

Figure 2: WVU power simulator in original configuration

\subsection{Addition of Digital Relays}

The first step in the upgrade was to install new digital relays donated by SEL, Schweitzer Engineering Laboratories, Inc. These are listed in table 1. The relays have been installed next to their electromechanical counter-parts and will be wired in parallel with them. This will allow either, or both, to be used if desired. Figure 3 shows these new relays mounted in the simulator. Three of the donated relays are yet to be installed the $300 \mathrm{G}$ generator relay and two of the 751 feeder protection relays. These will be reserved for use with the distributed generation equipment to be acquired later.

Table 1: List of equipment donated by SEL

\begin{tabular}{|c|c|}
\hline 4 & SEL 751 A Feeder Protection Relays \\
\hline 1 & SEL 387A Current Differential Relay \\
\hline 1 & SEL 300G Generator Relay \\
\hline 4 & SEL 351 Over-Current Protection Relays \\
\hline 1 & SEL 351S Protection Relay \\
\hline 1 & SEL 734 Revenue Metering System \\
\hline 1 & SEL 3351 System Computing Platform with Subnet Software \\
\hline
\end{tabular}




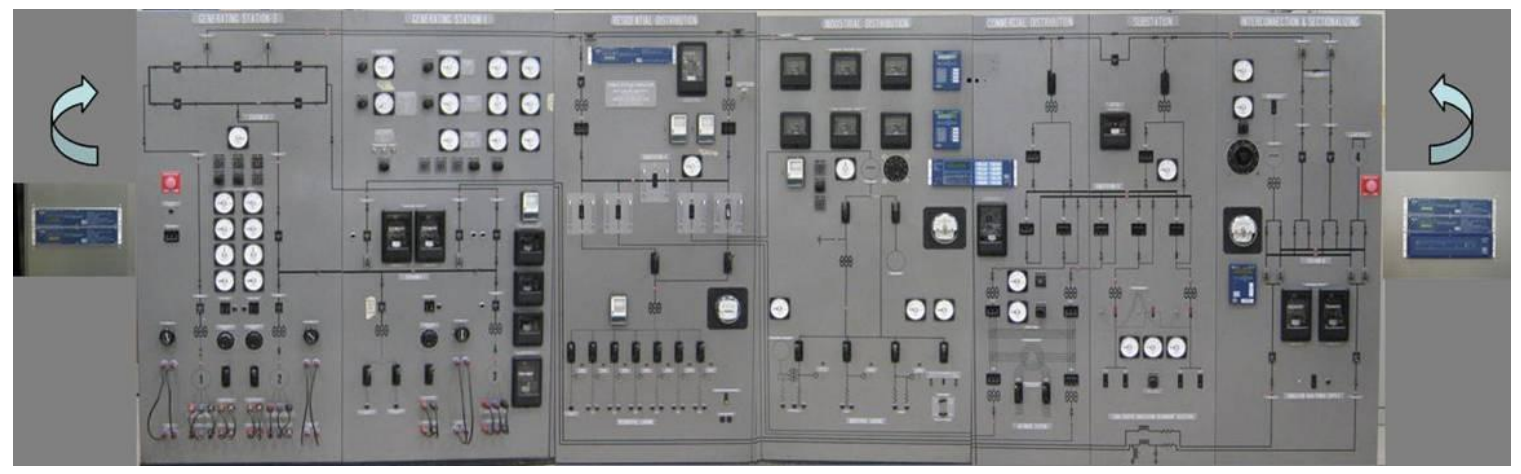

Figure 3: Power simulator with added digital relays

\subsection{Addition of Intelligent Electronic Switches}

The simulator has approximately 100 manually operated switches that allow power to be routed over different paths between the generators and loads. In stage two, the majority of those switches will be made redundant with micro controller switched optically isolated triacs. Each micro controller will also be paired with an ADE 7758 three-phase energy metering chip to monitor current and voltage at that node and to allow the MCU to focus on communications and higher level intelligence functions. All of the ADE7758 metering chips will be fed the same 10 $\mathrm{MHz}$ clock signal making it possible to synchronize waveform sampling across all nodes. When not needed the MCU controlled switches can be turned off and the simulator used in its original configuration. 


\section{Chapter 2 Literature Review}

\subsection{Digital Simulation of Power Systems}

Simulation of power systems is a critical component of predicting and thereby controlling their operation. Most power simulation is done digitally with models of varying complexity programmed in various computer languages FORTRAN, C, C++ etc. and in higher level mathematics programs, such as MATLAB. In this section a variety of digital simulators are described that cover this range from those written in a higher level language to those which run on hardware specifically designed to run simulations of this order.

\subsubsection{Power World Simulator}

Power World Simulator is a commercial stand alone power simulator that runs on Microsoft Windows 2000/XP/Vista machines. It's one of several power related software products from Power World Corporation. It can handle power flow for up to 100,000 buses and has a wide variety of add-ons available. It is a ready to go out of the box GUI based system. It can be used to simulate everything from straight forward power flow in an educational environment to economic analysis using some of its available add-ons. It uses a full Newton-Raphson algorithm and costs $\$ 8,000$ for the simulator alone, add-ons range from $\$ 1,500$ to $\$ 10,000$, but there are educational versions and free 12 bus evaluation versions available.

\subsubsection{PAT Power Analysis Toolbox}

PAT: Powers Analysis Toolbox is a Simulink toolbox written at WVU. Simulink is the graphical interactive component of MATLAB (2). Functions can be written and are "contained" within a particular block and then those blocks tied together to perform more complicated functions but retain their symbolic graphical nature to make interpreting the system much easier than trying to follow loops and functions in line by line program code.

PAT is a large set of custom blocks specifically designed to represent a wide variety of power system components that can be used in the Simulink environment to perform load flow, transient and small signal analysis of electric power systems. With the tool box, systems can be put together graphically making it much easier verify the layout of the model. The blocks are all 
pre-tested code which minimizes code verification, once code has been shown to work in one instance there is confidence that they will in every incidence.

Since PAT is a toolbox, it has a big advantage over dedicated digital power simulation packages in that other Simulink blocks can be written and used and the simulation environment expanded and adapted to simulate new hardware, try new control schemes and develop new and unique analysis tools. The disadvantage is that which is inherent in all modeling which is that the model only represents what is considered the important aspects of the physical system in order to be able to run the simulation in a short enough time to be useful. Even with the simplification of a model the run times usually far exceed real time reactions of the system, but with enough processing power, usually processors running in parallel real time analysis can be approached which is the claim of the next digital simulation example.

\subsubsection{RTDS Real Time Digital Simulator}

Real Time Digital Simulator is a digital simulator that claims to be capable of real time solutions to electromagnetic transients. It does this through parallel processing. Instead of a faster processor they have many doing calculations in parallel to find their solution. To do this special hardware specific software must be used. RTDS Technologies sells a modular hardware platform and the software that runs on it. They have several very specific user programs available that utilize the computational capabilities of their hardware, but for the most part their systems are setup to run closed loop testing of physical devices. That is they supply an input and sample and analyze the corresponding output. While this system may give results to particular simulations in "real time", they should not be confused with real time operating systems which refer to microprocessors running code where individual lines of code are repeated at the same clock cycle every time the code is run. This ensures repeatable performance in "real time", this is often done on the other end of the processor scale from the high speed parallel processing done in the RTDS system on small embedded processors in your washing machine for instance.

\subsection{Hardware Simulation}

All digital models have their limitations however due to the trade-off between model complexity and processing time. An alternative approach is to build systems using real components and test in a controlled environment. The advantage of this is that the system runs in 
real time and without the errors associated with an imperfect model. The trade for this level of realism is cost and limited flexibility, it is relatively easy to rearrange a system running as models on a computer, and it is much more costly and difficult to do in hardware. A computer may also be used to perform a variety of other tasks, where as the power simulator will probably not be useful for word processing.

\subsubsection{Shipboard APERC}

A hardware realization of a shipboard power system was made and reported on by members of APERC (2). The end result is a small scale lower power and voltage version of the US Navy's idea of an all electric ship including propulsion, simulated operational loads and weapons systems. The entire system is interconnected with a CAN bus that allows for multiagent intercommunication for testing reconfiguration and power management algorithms. The experience gained from this endeavor was the genesis of the power simulator upgrade described here.

\subsubsection{Hampden Engineering Corporation Power System Simulator}

The WVU power simulator was built by Hampden Engineering Corporation in the mid 70's. Hampden offers an upgraded version of the simulator with centralized computer control. This is probably the closest hardware available to the upgrade being performed at WVU. The new Hampden system the model \# 180 offers all of the features that came originally with the WVU system and has some upgraded digital components that can communicate with a central control computer, but does not have the distributed intelligence on the scale that the upgrade proposes. Without the MCU 's with their substantial communications capability at the large number of nodes testing of multi-agent and other distributed intelligence algorithms could not be explored, and since that is a major driver for the upgrade this option was not a viable alternative. Hampden was kind enough to send us new copies of our schematics however to replace several that had gotten lost over the years. It may also be of value to further pursue electronic copies of their modern system that we could modify to reflect the WVU upgrade. 


\section{Chapter 3 Power Simulator}

\subsection{Undergraduate Labs}

There are two undergraduate labs that are taught on the power simulator presently in the electromechanical energy conversion class EE335.

\subsubsection{Lab 4}

Lab 4 examines the unregulated voltage characteristics of a generator under different load conditions. A DC motor is used as the prime mover and its speed is set so the generator output is $60 \mathrm{HZ}$ under a no load condition. The generator field current is then adjusted until the output is 550 VAC. Load is then added, both balanced and unbalanced in turn and the voltage monitored while the frequency is kept a $60 \mathrm{~Hz}$.

\subsubsection{Lab 5}

In Lab 5 the students explore the concept of generators in parallel connected to an infinite bus. First a generator is brought up to speed and synchronized with the 3-phase power coming in from the local electricity provider and then an attempt is made at increasing the speed, this results in real power being added to the infinite bus of the local electricity provider but no change in speed. In the second half of the experiment the field current is changed while synchronized and a corresponding change in reactive power delivered to the infinite bus is observed. Since the real power stays virtually constant the result is a change in power factor.

\subsection{Web-Based Relay Management}

In 2008 a LDCSEE student, Brian Graeber completed his master's degree with a thesis titled "Web-Based Relay Management with Biometric Authentication" (2). The document is unfortunately not publicly available, but describes Brian's work implementing a thumb print reader to verify identity of personnel attempting to change relay settings remotely. This was implemented on two of the newly acquired and installed over current relays. With the advent of increased communication associated with the smart grid will come ever increasing concerns about cyber security. Cyber security will be a constant battle in the new smart grid and Brian's work is just the first of much that could be explored on the upgraded simulator. 


\subsection{Potential Research}

The power simulator has served a useful though, because of its age, a dwindling function for the department. With the upgrades both in place and pending it's believed that it could have a renewed life and be a major contributor on the smart grid front.

\subsubsection{Real Time Testing of MAS and Other Control Algorithms}

APERC has been doing research on using distributed intelligent agents with communication capability for several years as a solution to the communications bottleneck and information overload that are fundamental problems of the present day grid. This Multi-Agent System (MAS) approach has been simulated in software but real world hardware with its associated timing and throughput issues is needed to really explore the concept; the upgrade to the simulator was designed with this specifically in mind. The communication requirements and processing capabilities needed for studies on MAS were at the forefront of the hardware selection process. The fortunate side benefit is that the hardware is then also applicable to other ideas using immune system based algorithms for example as intelligence models for adaptive control of the grid.

\subsubsection{Reconfiguration}

One of the first problems to be addressed by the upgraded simulator will probably be the reconfiguration of the system due to an outage. With the intelligent connectivity available in the upgrade there will be ways to route power around faults and restore power to much of the system. MAS algorithms (2) would have direct applicability here as would the immune based approach (3). Once this problem has been explored the lessons learned from it can be applied to more subtle and complicated predictive control.

\subsubsection{Optimum Power Flow, Load Shedding and Distributed Generation}

With the ability to monitor load flow at virtually every node comes the ability to determine optimum power flow through the system as well as automatically prioritize and shed load when needed or bring on distributed generation to abate a critical condition all using the distributed intelligence built into the system. These are all ideas that could be explored with the simulator that could have a tremendous impact on the grid of the future. 


\subsubsection{Communication Experimentation}

Communications is a critical component of any distributed intelligent system and models have shown that a multilayered communication scheme can offer a dramatic improvement in communication integrity and throughput. The MCU's chosen to act as the distributed intelligence were picked in large part for their ability to communicate via many distinct ports, Ethernet, Can bus, and RS-232 that could be used as wireless ports are all available to the algorithm explorer.

\subsubsection{Scalability}

Recently Summiya Moheuddin, also with APERC defended her master's thesis and published a paper on the optimization of the number and placement of distributed agents in a system that is scalable meaning the algorithm employed to pick the placement and number was unaffected by the size of the system (4). Her work could be implemented and verified on the new system and could also have great impact on the evolving smart grid.

\subsubsection{Communication Security}

As indicated earlier cyber security is a very large concern in the new grid and the upgraded simulator has been designed with this in mind. A very attractive feature of these particular MCU's is that they handle cryptographic processing not with the main processor but with a dedicated cryptographic co-processor. The MCU's were designed specifically to be used in a secure mode and there is no processing capability lost by implementing state of the art security measure 


\subsection{SEL Visit}

\section{Chapter 4 Digital Relay Installation}

When it was decided that the power simulator could be upgraded, Derek Ashby the local Schweitzer Engineering Laboratories, Inc. (SEL) representative was contacted to inquire about purchasing a few digital relays that could be used for experimentation. Derek brought along Lee Underwood an applications engineer for SEL and they were able to get 12 relays and a computing platform donated from SEL at no cost, see Table 2. The new relays were chosen by Lee to represent the modern equivalent of the electromechanical relays in the original system.

Table 2: Original electromechanical relay vs. SEL digital counterpart

\begin{tabular}{|c|c|}
\hline Original Electromechanical & Donated Digital Counterpart \\
\hline Type CO-8 Over-Current Relay & 4 SEL 751A Feeder Protection Relays \\
\hline $\begin{array}{c}\text { Type CA Percentage Differential } \\
\text { Transformer Relay }\end{array}$ & 1 SEL 387A Current Differential Relay \\
\hline Type CRN-1 Relay & 1 SEL 300G Generator Relay \\
\hline Type CR 2 Directional Over-Current Relay & 4 SEL 351 Over-Current Protection Relays \\
\hline Type CW Power Relay & 1 SEL 351S Protection Relay \\
\hline Mark I Demand Register & 1 SEL 734 Revenue Metering System \\
\hline
\end{tabular}

\subsection{Installation}

The original system was intended to be a complete system, so there was very little room for addition of the new hardware, see Figure 4. 


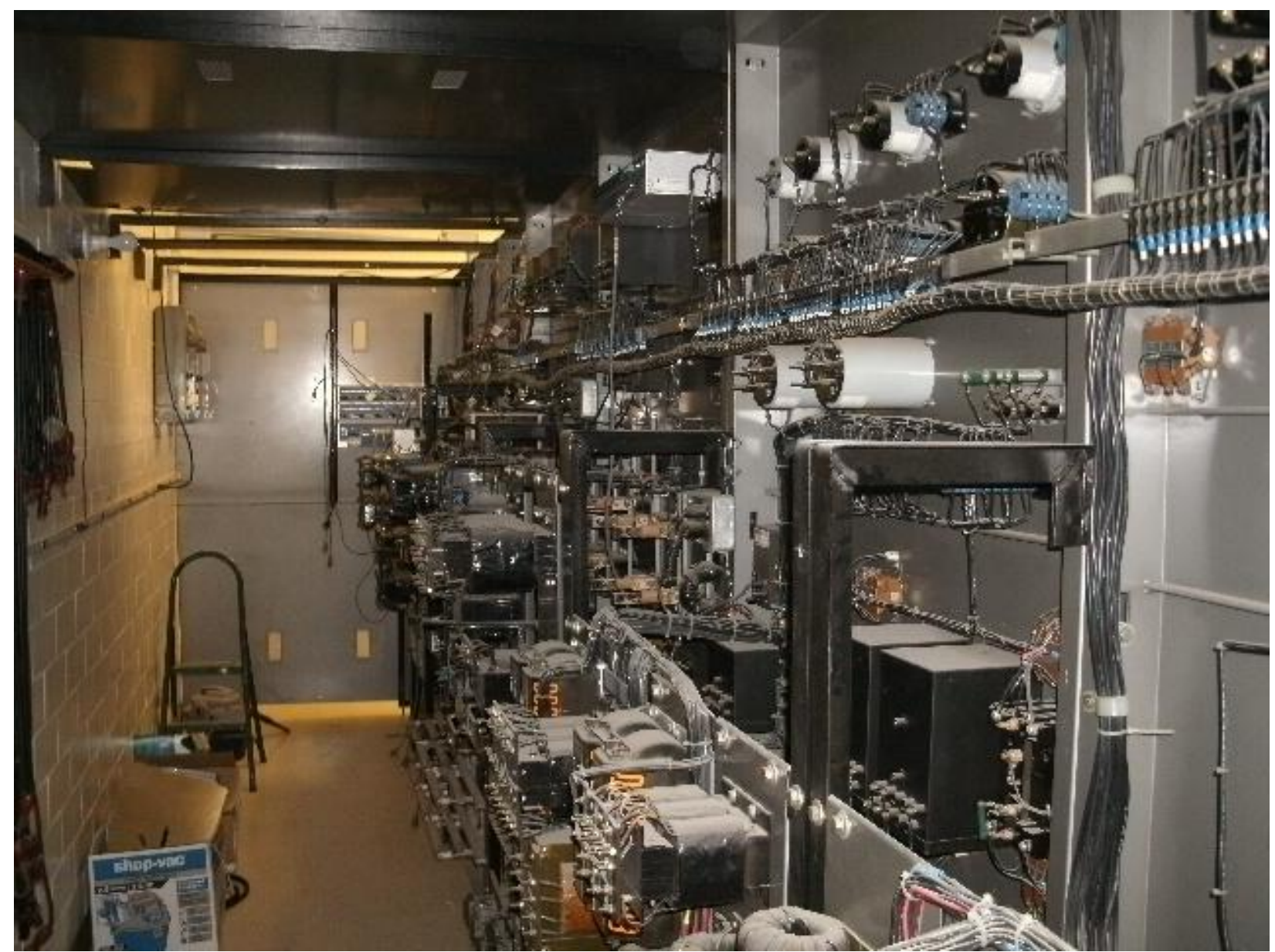

Figure 4: A peek behind the curtain at the back of the power simulator front panel

After careful planning, most of the relays were fit into the panels relatively close to their electromechanical counter parts. In one case a strut had to be cut and lengthened to retain the physical integrity of the simulator see Figure 5. 


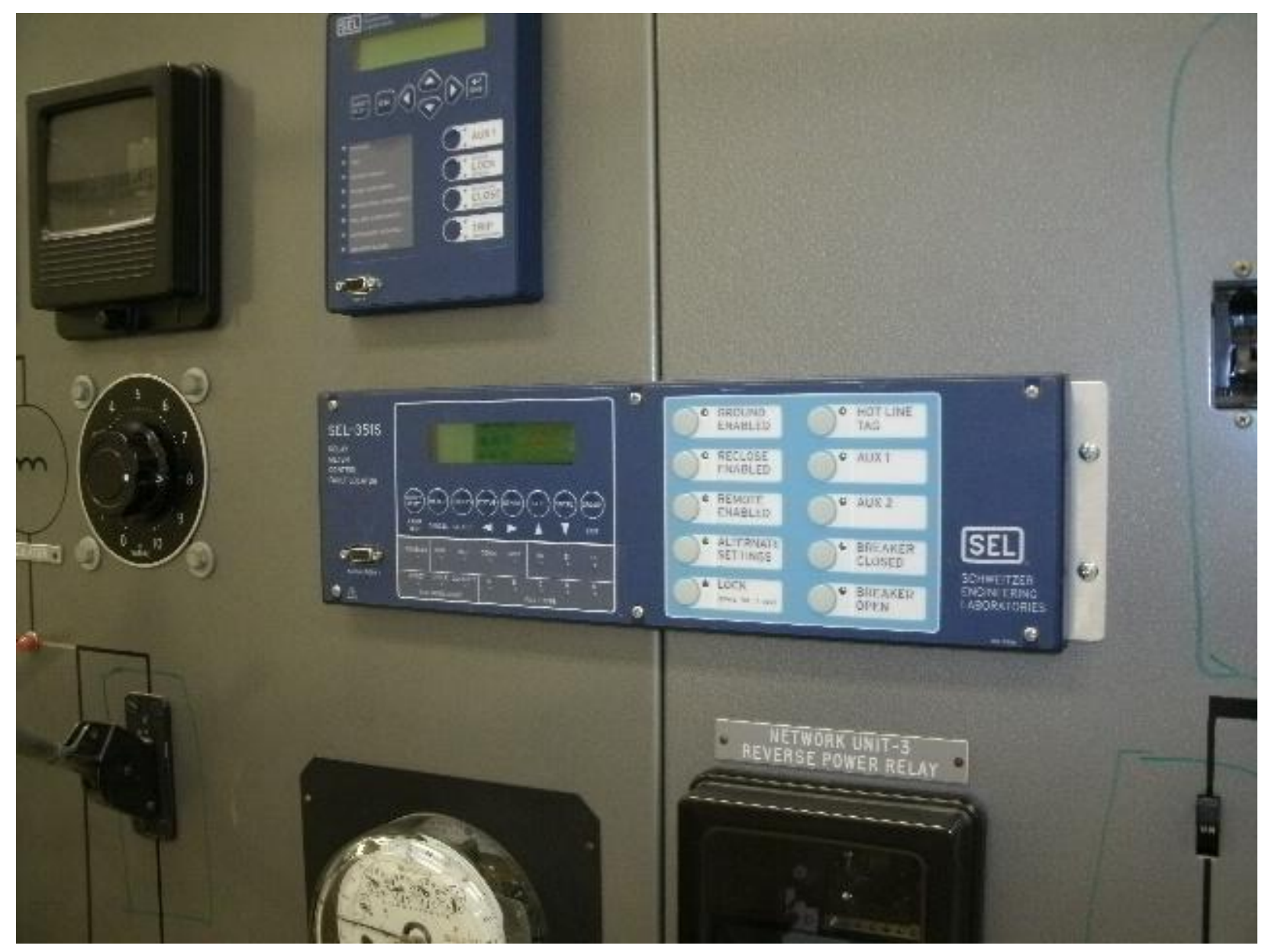

Figure 5: SEL 351S the most difficult relay installation 


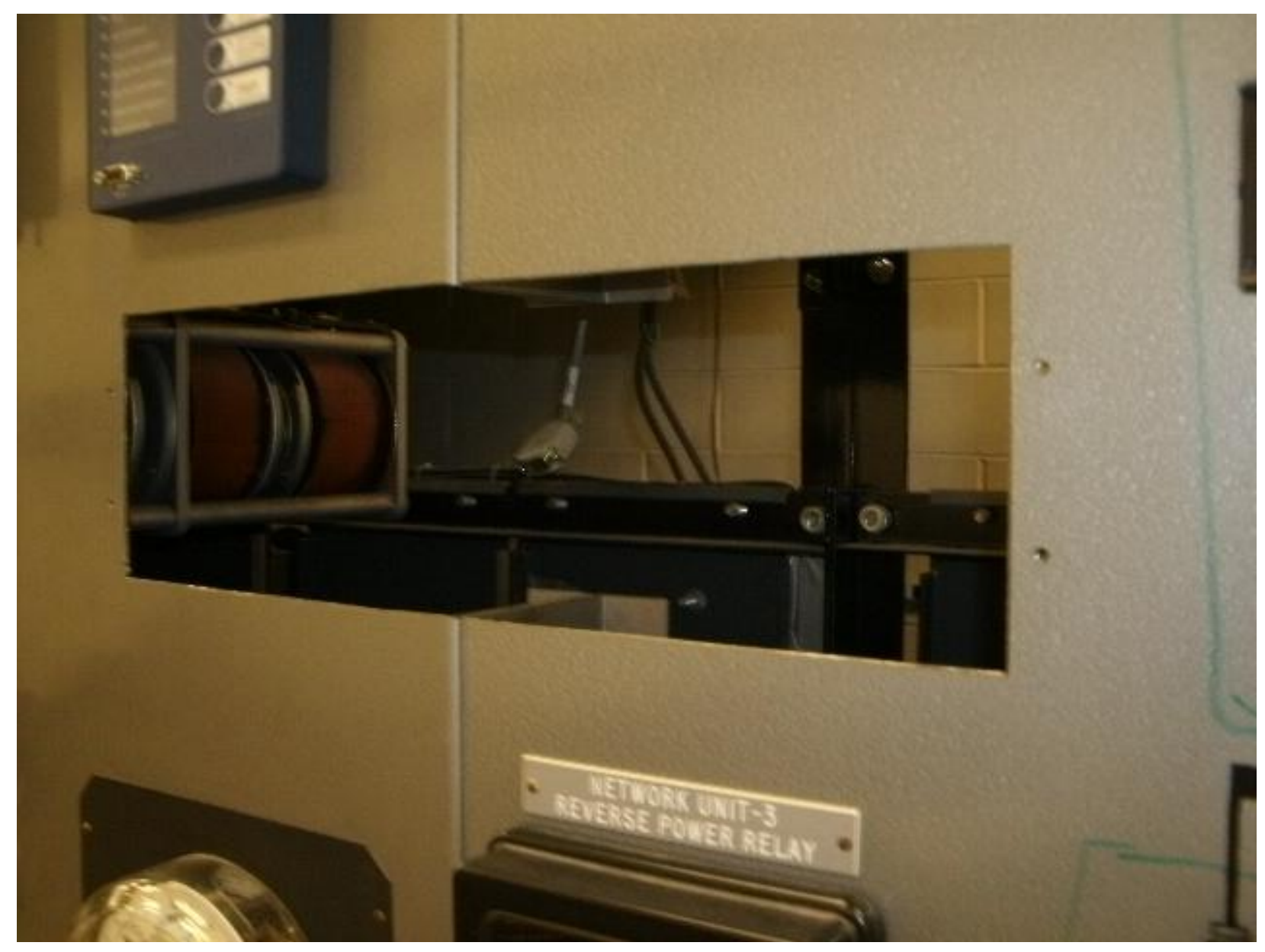

Figure 6: Panel junction that had to be cut for installation 


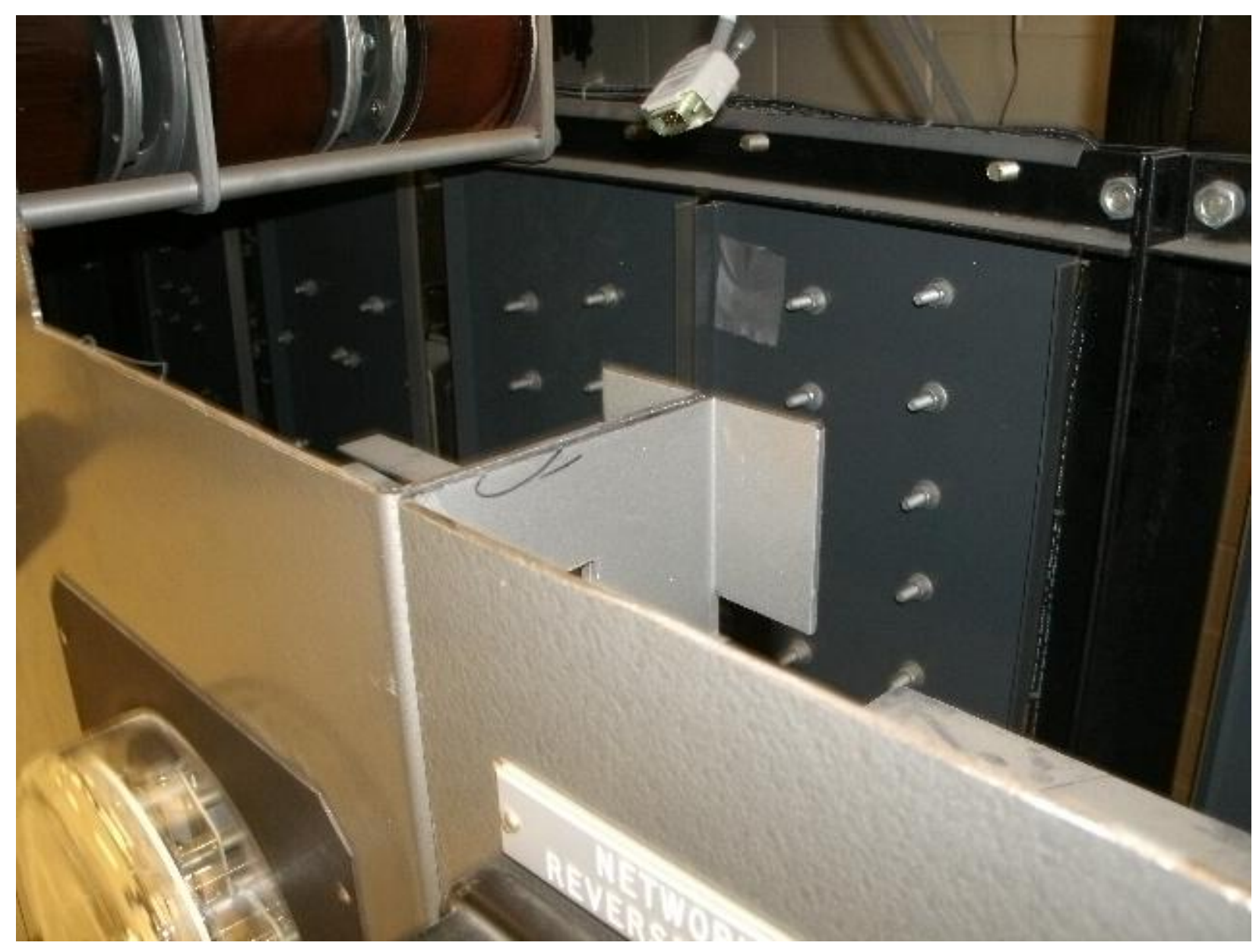

Figure 7: Panel junction close-up 


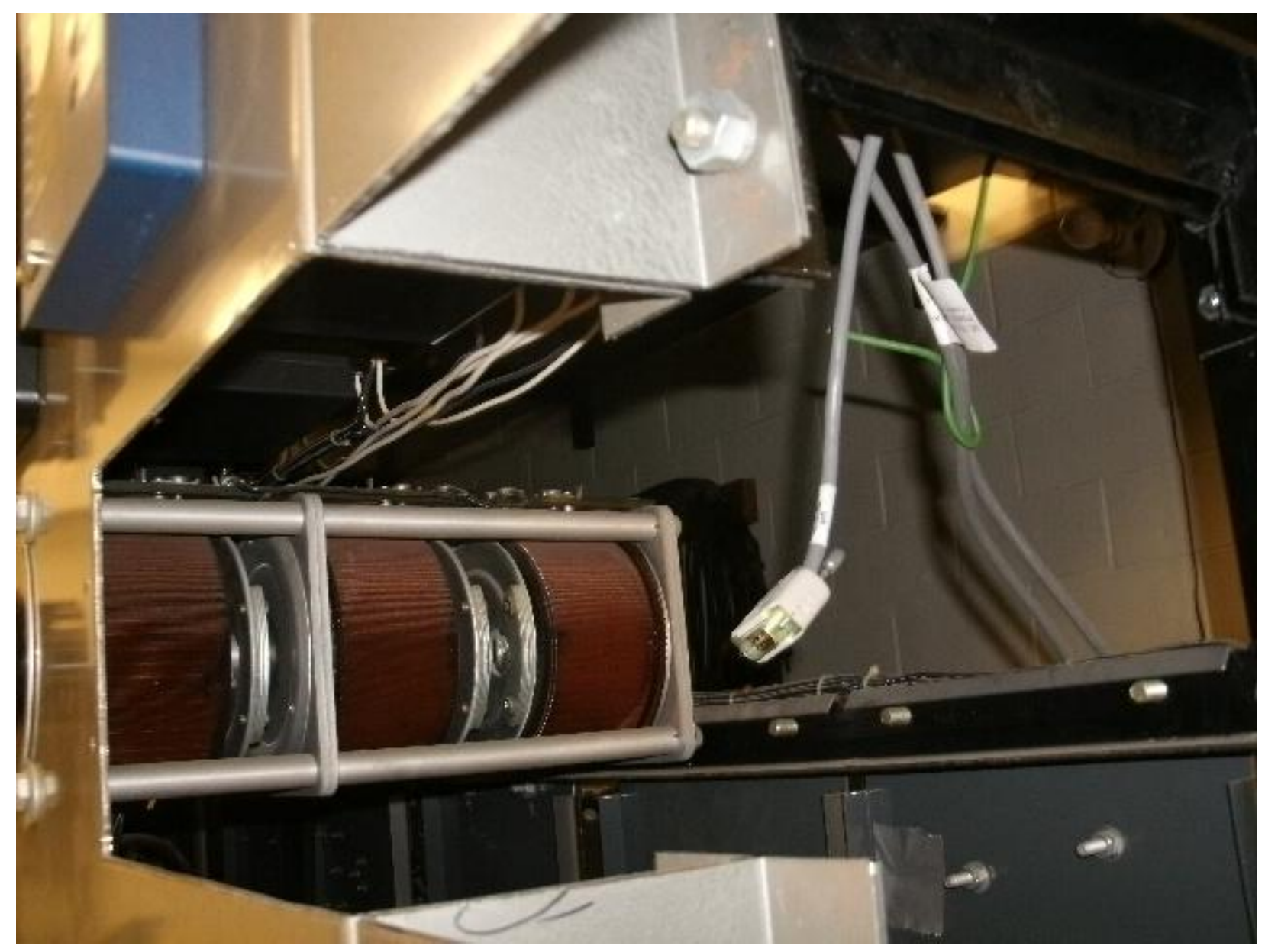

Figure 8: Panel junction with new strut bolted in place 


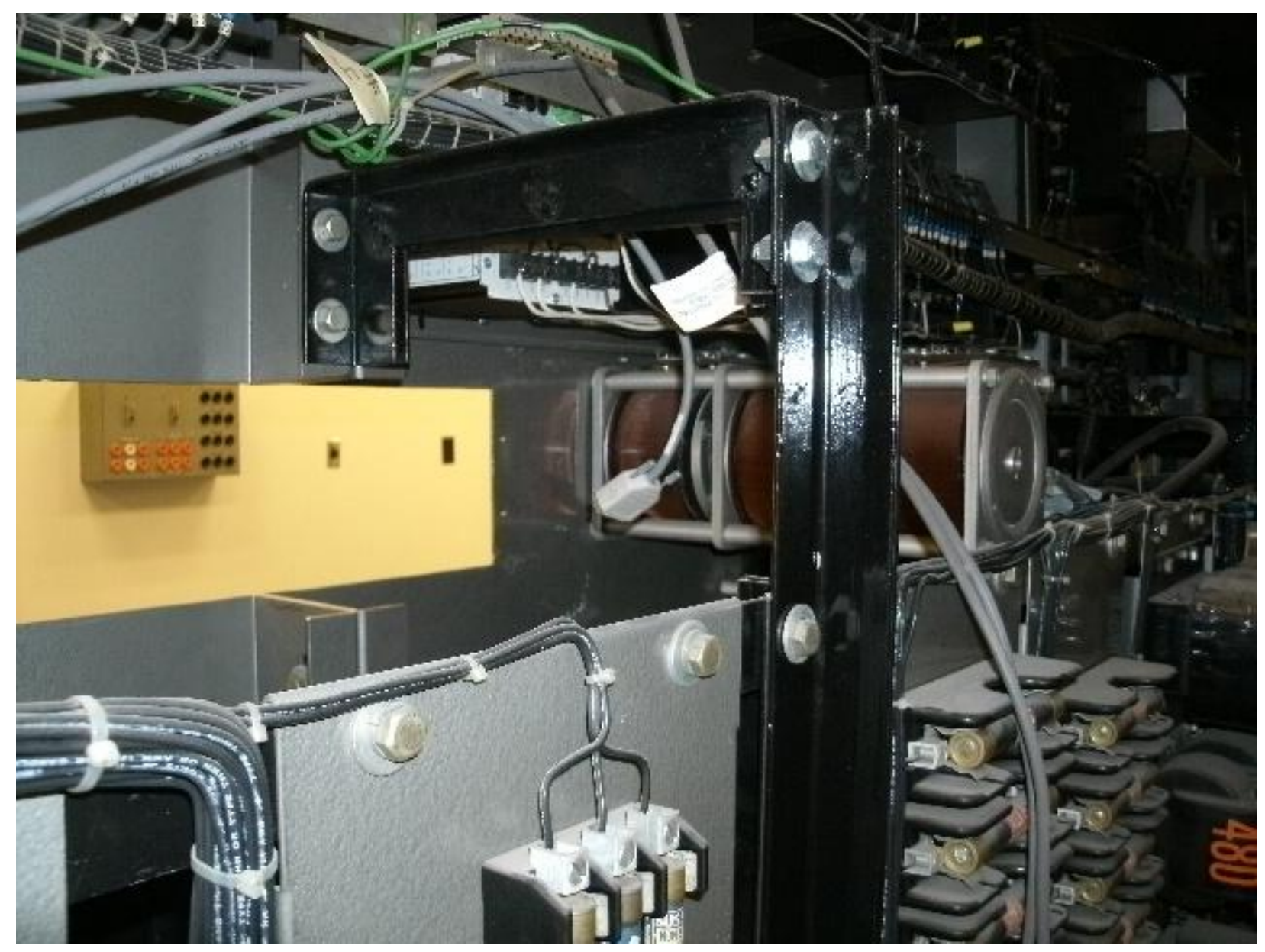

Figure 9: View of extended strut from behind the panel

In another case the strut had to be shortened see illustrations 9 and 10. Additional reinforcement was added to the panels on the ends because they are of a lighter gauge of sheet metal and the openings cut into them needed to be large to accommodate two and three devices, illustrations 9, 10 and 11. 


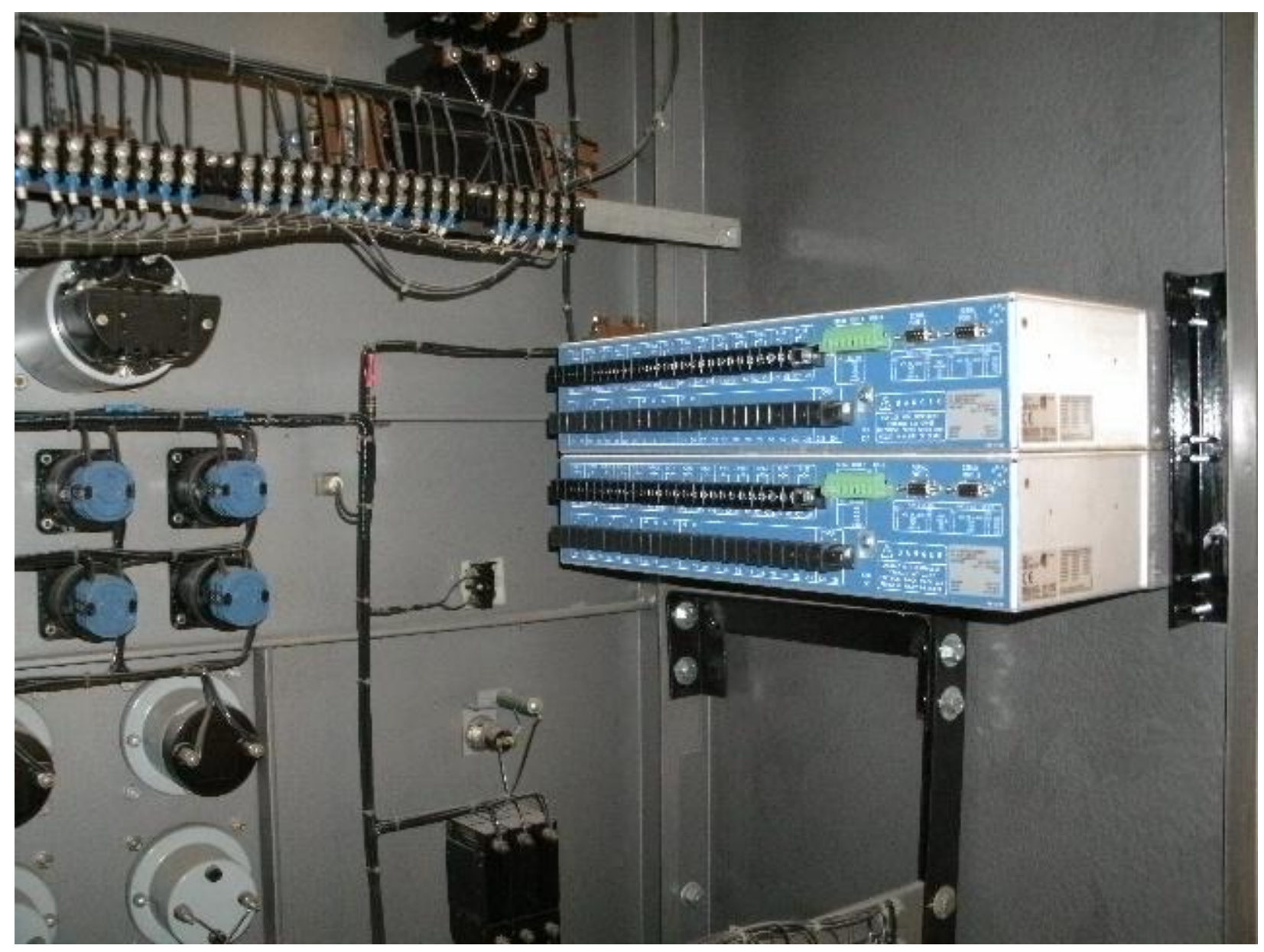

Figure 10: View of shortened strut and end panel reinforcement

Three of the donated relays were not installed. These were the $300 \mathrm{G}$ generator relay and two of the 751 feeder protection relays. The $751 \mathrm{~s}$ were chosen because they were redundant and they are the most basic relay so are an excellent choice for students to work with on the bench to become familiar with the equipment. The $300 \mathrm{G}$ had no electromechanical counter-part and is intended to be used with the distributed generation equipment to be acquired later.

The installed751 feeder relays have been wired for current monitoring and used in another thesis by Brian Graeber. Using the wiring diagram for the electromechanical relay it is pretty straight forward to wire up the new digital relay in the same configuration. The new relays have more capability but to access that capability additional sensors are required. In the case of the 751's mentioned in addition to current sensing the digital relays have the ability to monitor voltage as well. This had posed a problem until recently when a complete set of schematics for 
the simulator was acquired from the manufacturer. With the information from these diagrams the full capability of the new relays should be able to be fully utilized. 


\section{Chapter 5 Intelligent Electronic Switch Hardware Selection}

The digital relays bring the micro grid up to a current state of the art level, but to allow for advanced reconfiguration algorithm testing automation of the manual switches on the simulator is desired. It would be possible to use relays for this function except the size and cost of the system would be prohibitive. The solution was to design a compact solid state switch that could be controlled by a micro-controller that would also sample current and voltage at that node.

\subsection{MCU Selection}

A search was made to find a compact micro controller that gave as much processing and communication capability as currently available to provide the algorithm developers as large a canvas to try their ideas on. A demo board from Freescale, the M52259DEMOKIT was chosen. It has a 32 bit $80 \mathrm{MHZ}$ processor with 512 Kbytes of flash memory, 64Kbytes SRAM, and a cryptographic accelerator unit to allow for secure communication without taxing the main processor. It comes with two USB ports, one 10/100 Ethernet port, an RS-232 port and a high speed CAN bus port all in a 3"x3.5" two board package for $\$ 49$. Figure 3 shows the demo board with its communication ports. 


\section{M52259DEMOKIT Block Diagram}

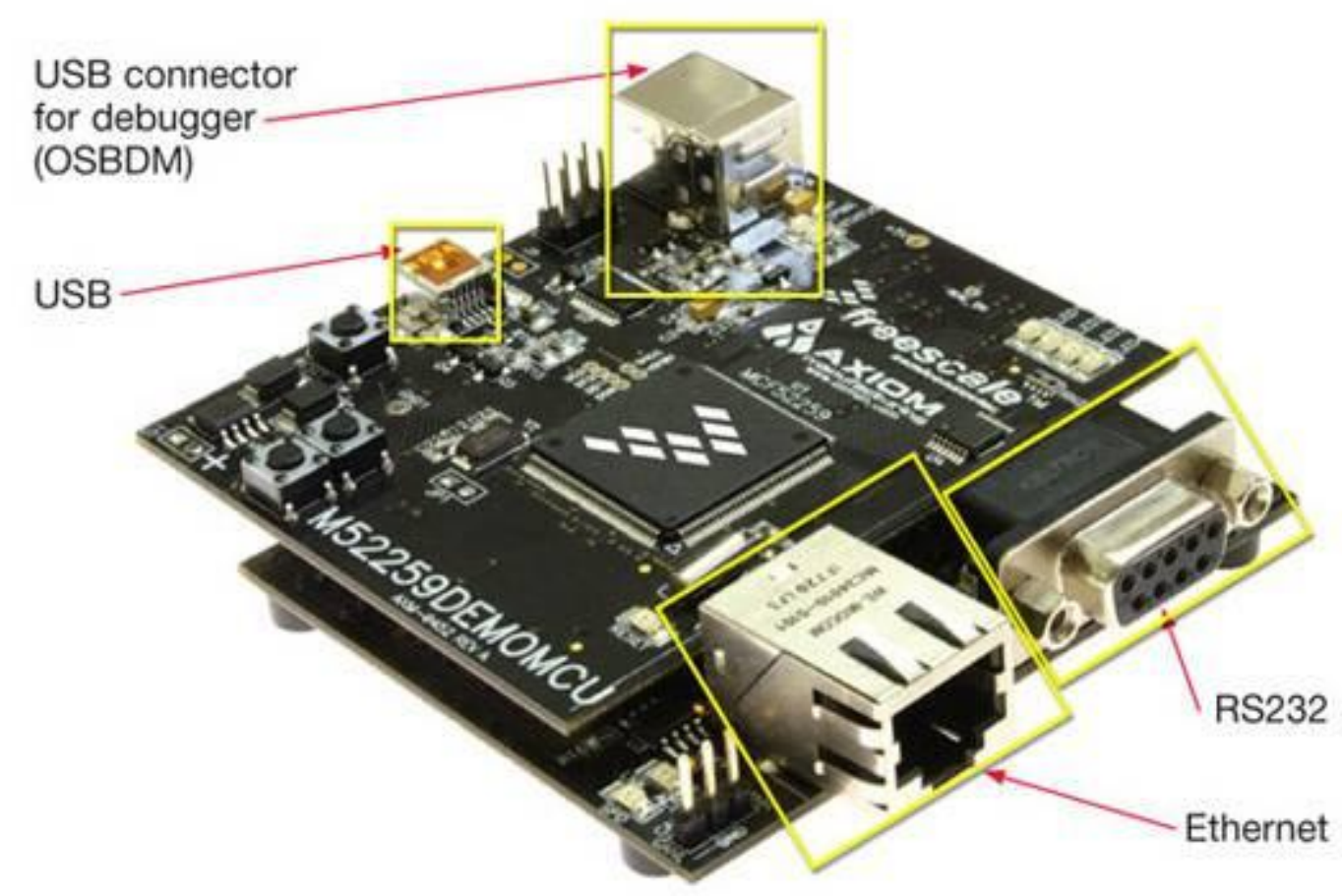

Figure 11: Freescale M52259DEMOKIT

\subsubsection{Communications Capability}

From the beginning of this project it was determined that communication was a high priority. This came directly from the desire to test MAS ideas and from the input of Dr. Reynolds involving multiple communication paths to insure data integrity. The single biggest reason this particular demo board was selected was the multiple communication ports available. Both CAN bus and Ethernet can be used for a multiple wired communications scheme and the RS-232 port can then be dedicated to a wireless module. This still leaves the on board diagnostics USB port available for programming and the QSPI for interface with the ADE7758 meter chip. It is believed that the remaining USB port could be used for a memory device and that still leaves an $\mathrm{I}^{2} \mathrm{C}$ interface available for future use.

\subsubsection{Processor}


In addition to communications it was also apparent early on that security will be a very big issue. To address this issue the processor comes with a dedicated cryptographic co processor specifically to handle secure communication.

\subsubsection{Software}

Freescale offers software to be used in programming their devices. The limited trial version appears to be sufficient for initial programming although the full version may be needed in the future.

\subsection{Triac and Opto-Isololator Selection}

The power simulator can have voltages as high as $750 \mathrm{VAC}$ and currents in the 5 ampere range. The triacs selected are of the snubberless type and rated for 8 amps at 1000VAC in order to give a comfortable safety margin. The triacs are driven by opto-isolators specifically designed to work with triacs. Non-zero crossing devices were chosen. Since all three phases will be switched at the same time it seemed that zero crossing switching might pose a problem during turn on.

\subsection{Metering Integrated Circuit Discovery and Selection}

Initially the idea was to use the A/D capability of the MCU to sample current and voltage at the node. While searching for triacs and opto-isolators a multifunction energy meter IC was discovered that would perform those functions and many more leaving the MCU with more processing and communications capability. The specific one chosen the Analog Devices ADE7758 is a three phase high accuracy energy measurement IC. It has a SPI interface and a wide variety of relay type features making it an ideal if not serendipitous find.

\subsection{Block Diagram}

All of these parts fit together in a straightforward module as shown in the block diagram of Figure 12. 


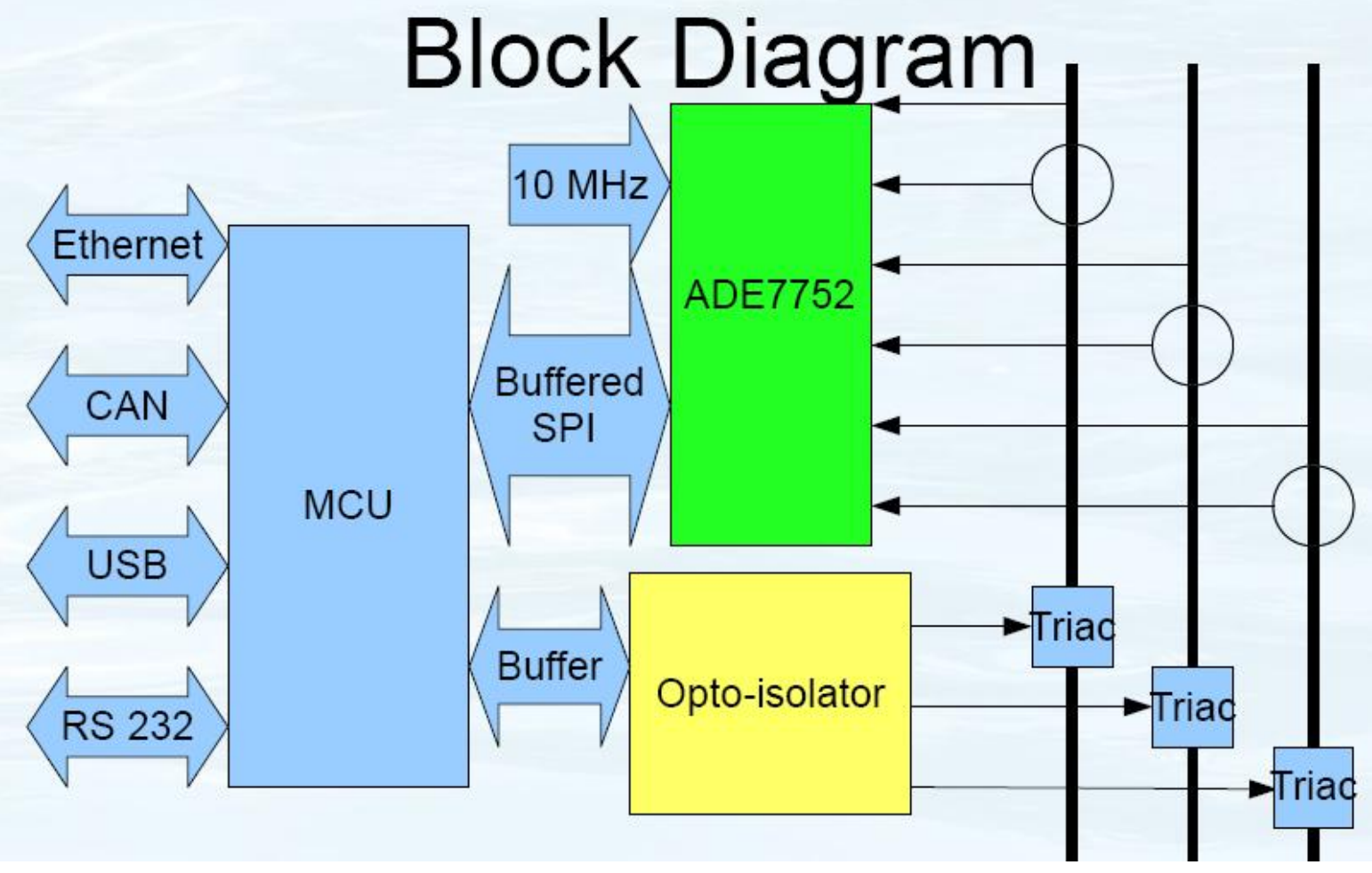

Figure 12: Block diagram of intelligent electronic switch 


\section{Chapter 6 Circuit Design and Board Fabrication}

The ADE7758 is only available in a surface mount package. This necessitates fabrication of a printed circuit board and surface mount soldering techniques. A design was drawn up and sent out for fabrication and a surface mount soldering station assembled and tested.

\subsection{Metering Circuit}

The ADE7758 Poly Phase Multifunction Energy Metering IC has built in analog to digital converters for sampling all three phases in both voltage and current. The input of the $\mathrm{A} / \mathrm{D}$ 's is $-0.5 \mathrm{~V}$ to $+0.5 \mathrm{~V}$ so the line voltages must be scaled down to that range with voltage dividers.

Traditionally current is sensed with a current shunt of some sort often with an isolation transformer to step down the current. Effectively what this does is convert the current to a proportional voltage that voltage is sample and the corresponding current derived from the amplitude of the voltage. Alternatively a Hall Effect sensor could be used but they are in the $\$ 80$ range and a bit too large for this project. The ADE7758 can be used in the normal current shunt mode but the engineers at Analog Devices have also come up with a unique alternative solution.

The voltage induced in the secondary of a transformer is proportional to $\mathrm{dI} / \mathrm{dT}$ of the primary. The constant of proportionality is the mutual inductance between the primary and the secondary. Since this circuit is really only set up for signals with no DC component the engineers decided that the secondary voltage could be sampled and then mathematically integrated to determine the current though the primary. An advantage of this is that there is almost no power that has to be dissipated in the secondary circuit.

As in all analog to digital conversion anti-aliasing filters need to be used. The circuit recommended by Analog Devices was used and a copy taken directly from the data sheet is shown here (2). 


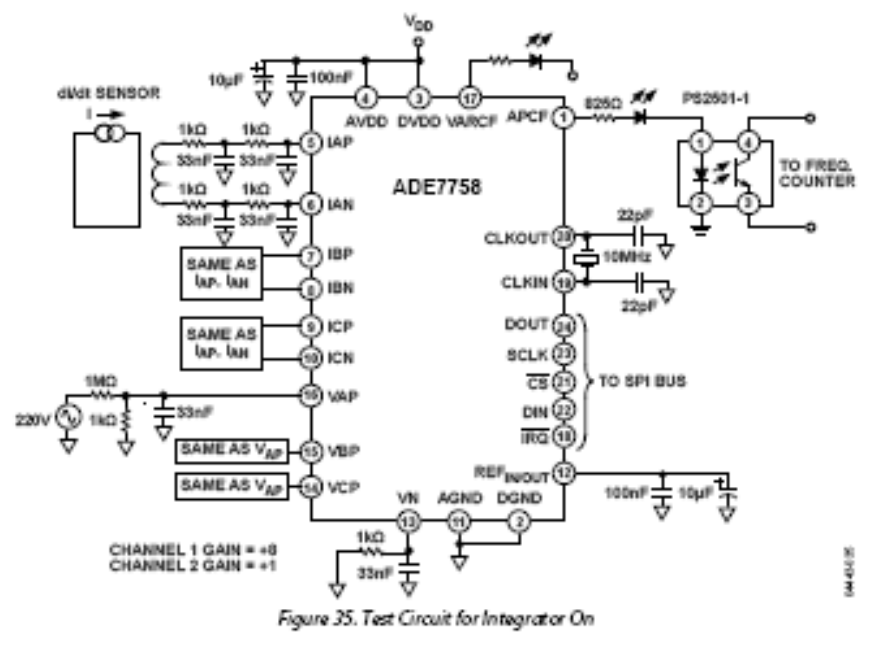

Figure 13: Circuit recommended by Analog Devices (2)

The output circuits at pins 1 and 17 were not installed nor were the capacitors for the clock circuit but the rest of the circuit was used as shown.

The 5VDC required by the IC comes directly from a regulator on the printed circuit board. This ensures that the IC receives a stable voltage. Since the M52259DEMOKIT also takes 5VDC, they can both be supplied by the same source set so the voltage at the boards is above the $5.5 \mathrm{~V}$ needed for ample headroom at the regulator. The regulator chosen only supplies 50mA but the ADE7758 only draws $21 \mathrm{~mA}$ maximum.

\subsection{Level Shifting and Buffering}

The M52259 microprocessor runs on 3.3VDC, unfortunately the ADE7758 runs on 5VDC causing a problem when the two are setup to talk to each other. The specifications for the input to the ADE7758 are within the output range of the M52259, so no level shifting is needed there. The input to the M52259 is not specified to handle the logic levels supplied by the 5 volt ADE7758 so a level shifter 74LVC08AD was used between the two.

\subsection{Triac Triggering}

Only three of the four gates are needed for level shifting leaving a spare gate which can be used as a buffer between the MCU and the opto-isolators see Figure 14. The output of the level shifter is 50mA per gate. The opto-isolators are wired in parallel to stay under the 3 VDC supplied by the M52259DEMOKIT and draw 10 to $20 \mathrm{~mA}$ each. $20 \mathrm{~mA}$ was chosen as the 
operating load so the combined draw is about $60 \mathrm{~mA}$ and beyond the range of the level shifter. A simple transistor circuit composed of a 2N2222 NPN small signal transistor running in saturation when on and a couple of $10 \mathrm{ohm}$ current limiting resistorsR 1 and R2 was used a as a current amplifier to ensure all parts were within their design limitations.

The triacs need a minimum gate current to turn on and that is determined by gate resistors $\mathrm{R} 3$ for phase $\mathrm{A}$ etc. In order to get the largest voltage range for the triac a gate resistor was chosen that would give a bit less than the recommended maximum gate current of about $100 \mathrm{~mA}$ for the maximum voltage on the line. 750VAC was selected as the maximum and 75mA as the largest RMS current desired. A $10 \mathrm{~K}$ ohm resistor in the gate circuit then limits the gate current to about $75 \mathrm{~mA}$. This should allow the triacs to work down to about 180VAC typically based on the typical values for gate trigger current, but their value may have to be adjusted if the operating voltage is used in that range because the triacs are specified to work at a 50mA max gate trigger current which corresponds to 500VAC

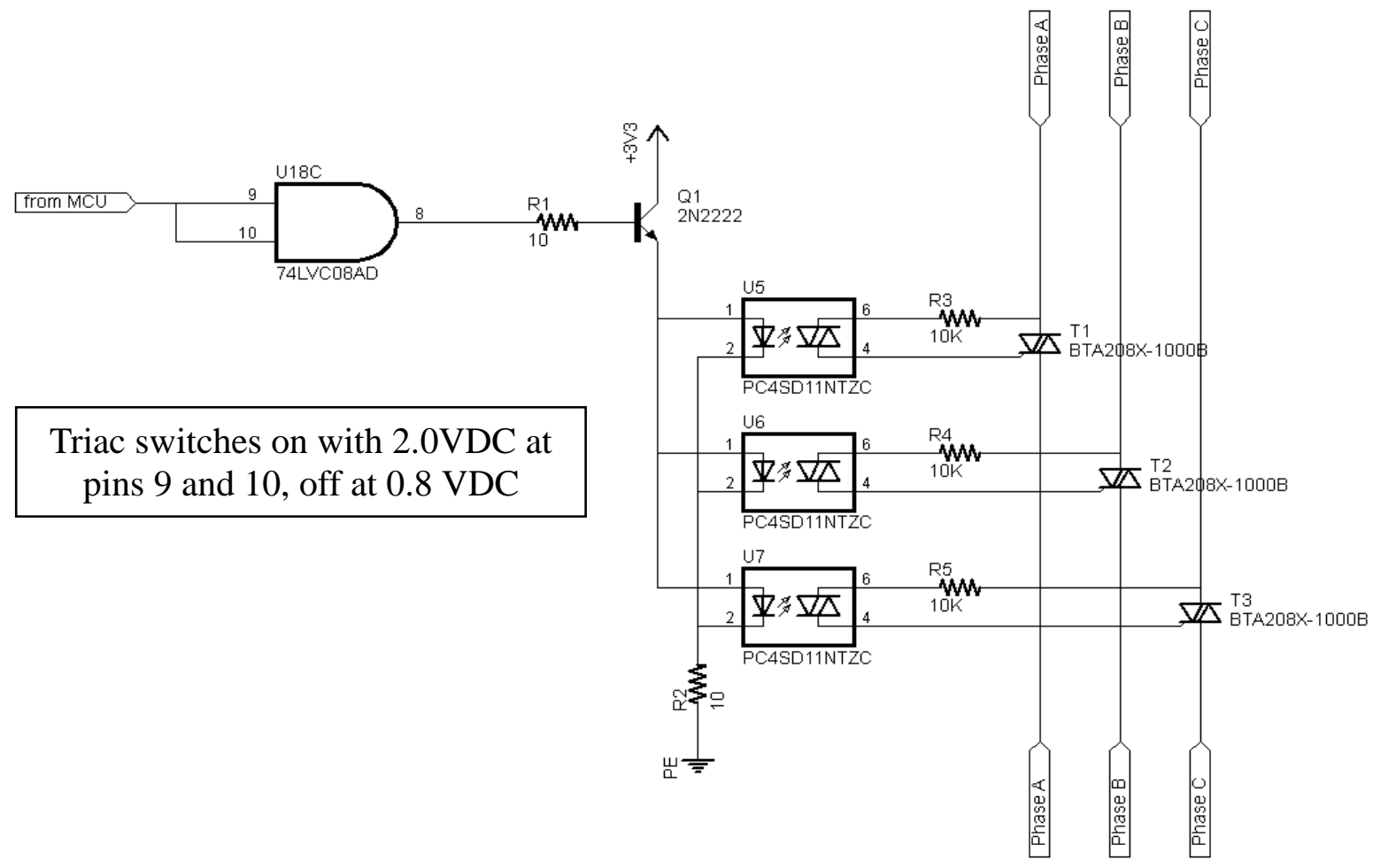

Figure 14: Triac triggering circuit 


\subsection{Fabrication}

The circuit boards were drawn up using Sunstone Circuit's PCB $123{ }^{\circledR}$ CAD software and the boards were then fabricated by them see Figure 15. This was effectively a prototype so the board was designed with the ability to change the layout to accommodate minor changes. There are places in the triac part of the circuit where snubber circuits can be added if they are found to be needed later. The level shifter is left disconnected with solder pads so that it can be reconfigured. Parts of the meter chip like the clock and SPI interface are left open with solder pads. With this setup the clock signal can be supplied by an external clock or an individually dedicated one may be used.

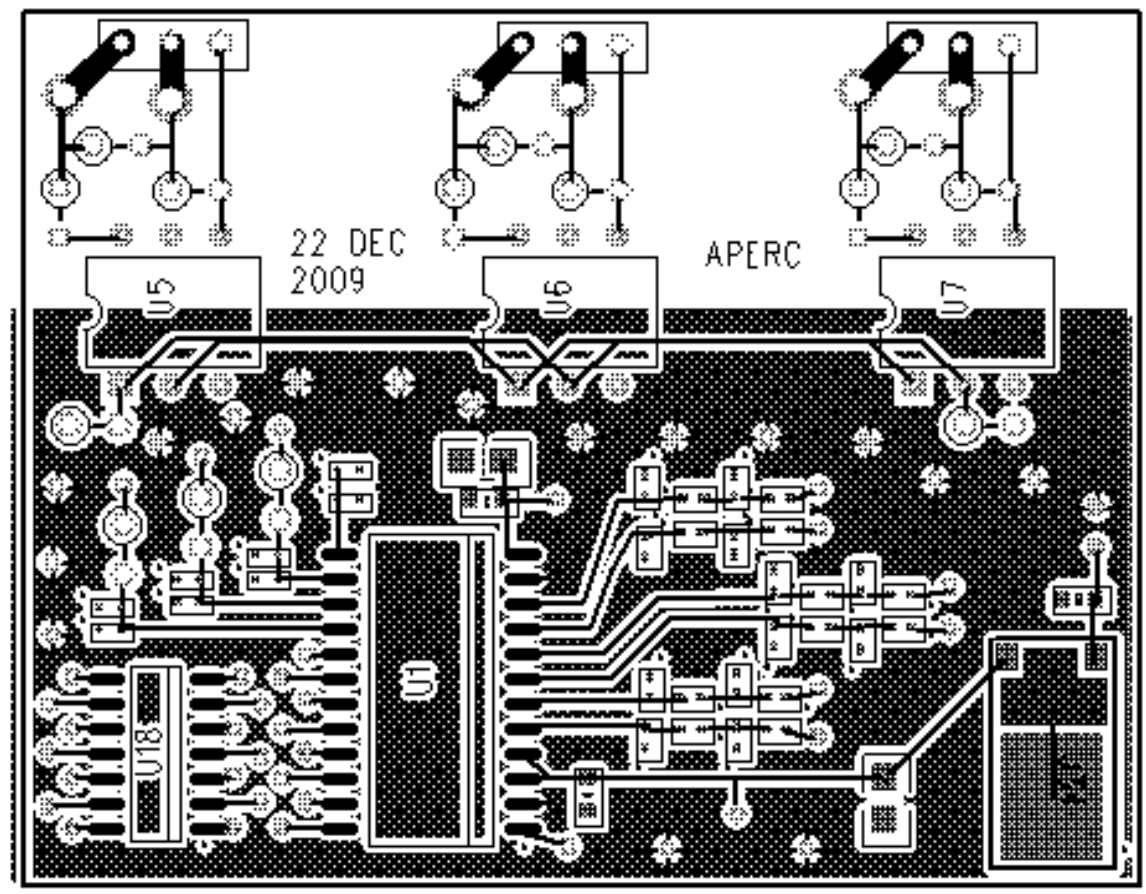

Figure 15: Sense and control printed circuit board layout

\subsection{Surface Mount Soldering}

Figure 16-Figure 21 shows the surface mount soldering process. 


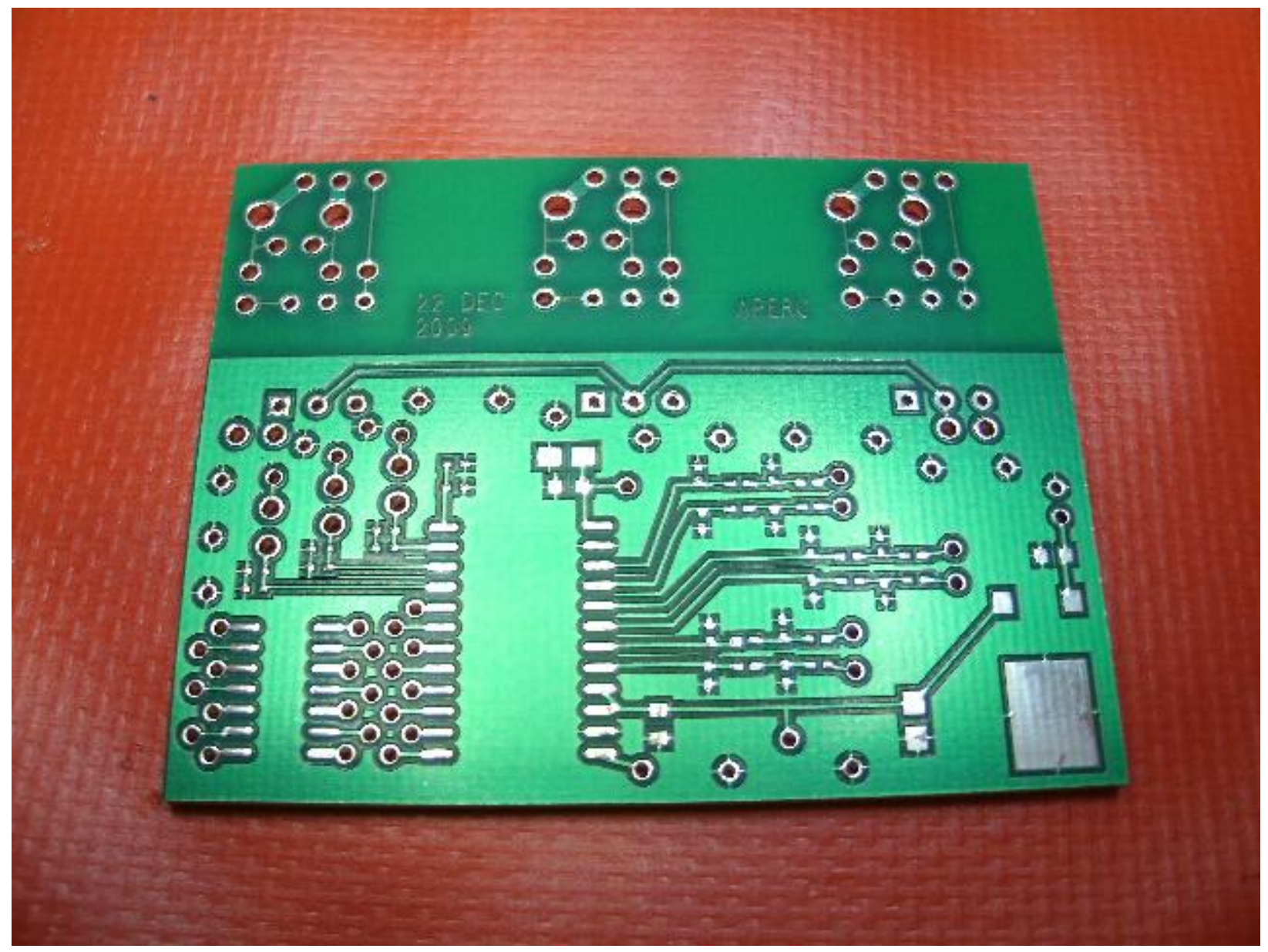

Figure 16: Bare printed circuit board ready for surface mount soldering; the transparent green coating is the solder mask.

Solder paste, which is small beads of solder suspended in a viscous flux paste needs to be kept refrigerated in order to slow the eventual separation. It is applied by syringe to the pads of the individual components, and then the parts are placed on the solder paste which holds them temporarily in place until the whole board is heated, melting the solder and bonding the parts to the pads. 


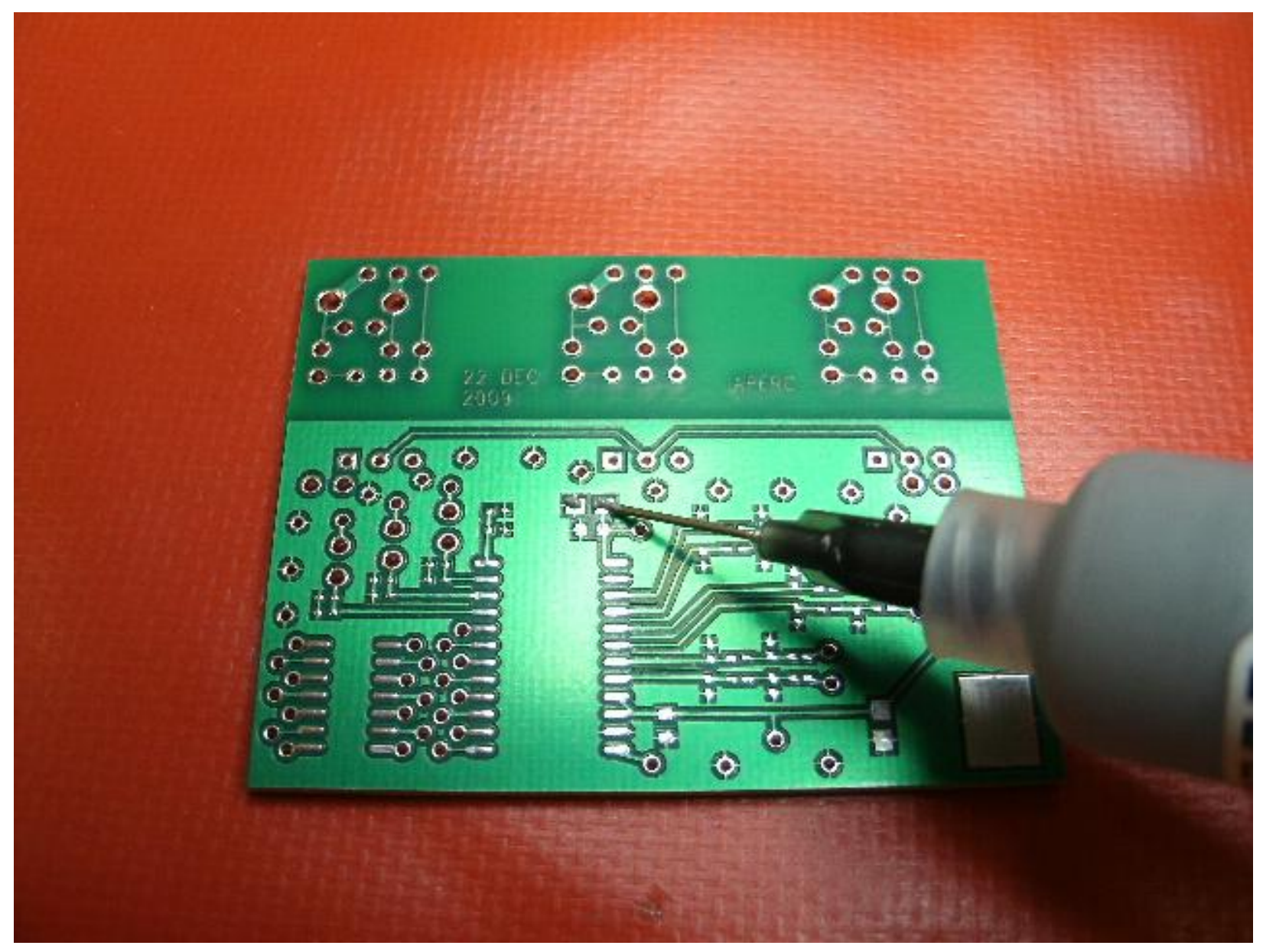

Figure 17: Applying solder paste to the solder pad of one of the large capacitors 


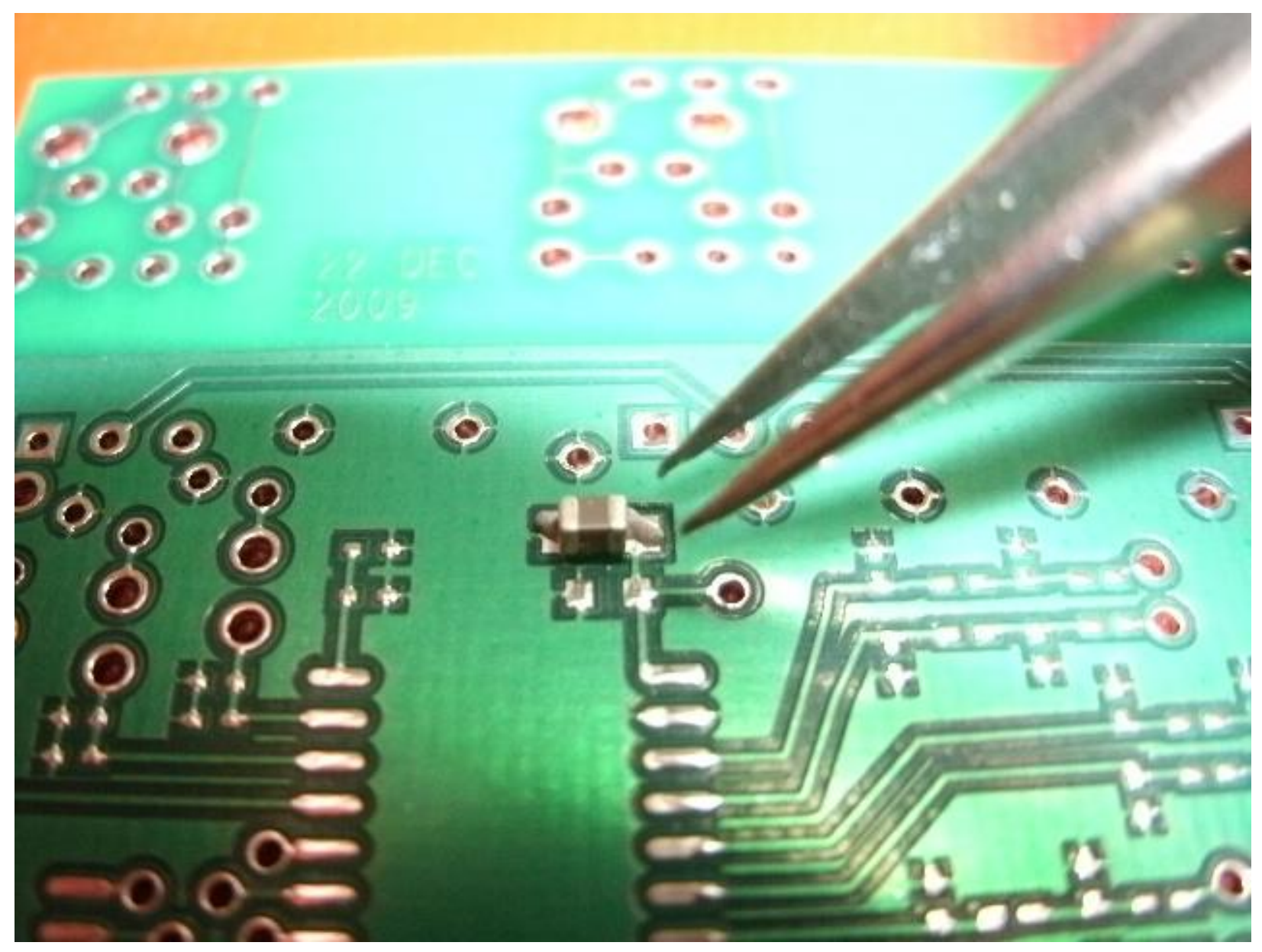

Figure 18: Placing the capacitor on it's solder pads with solder paste in place

It seems to work best if all of the smallest parts are placed first then the next largest etc. For the large multi-pin chips a bead can be run along the pads without worrying too much about getting the solder paste in between the solder pads. When heated to its melting point the cohesion of the molten solder and its lack of adhesion to the solder mask will make it bead up over the appropriate pads. 


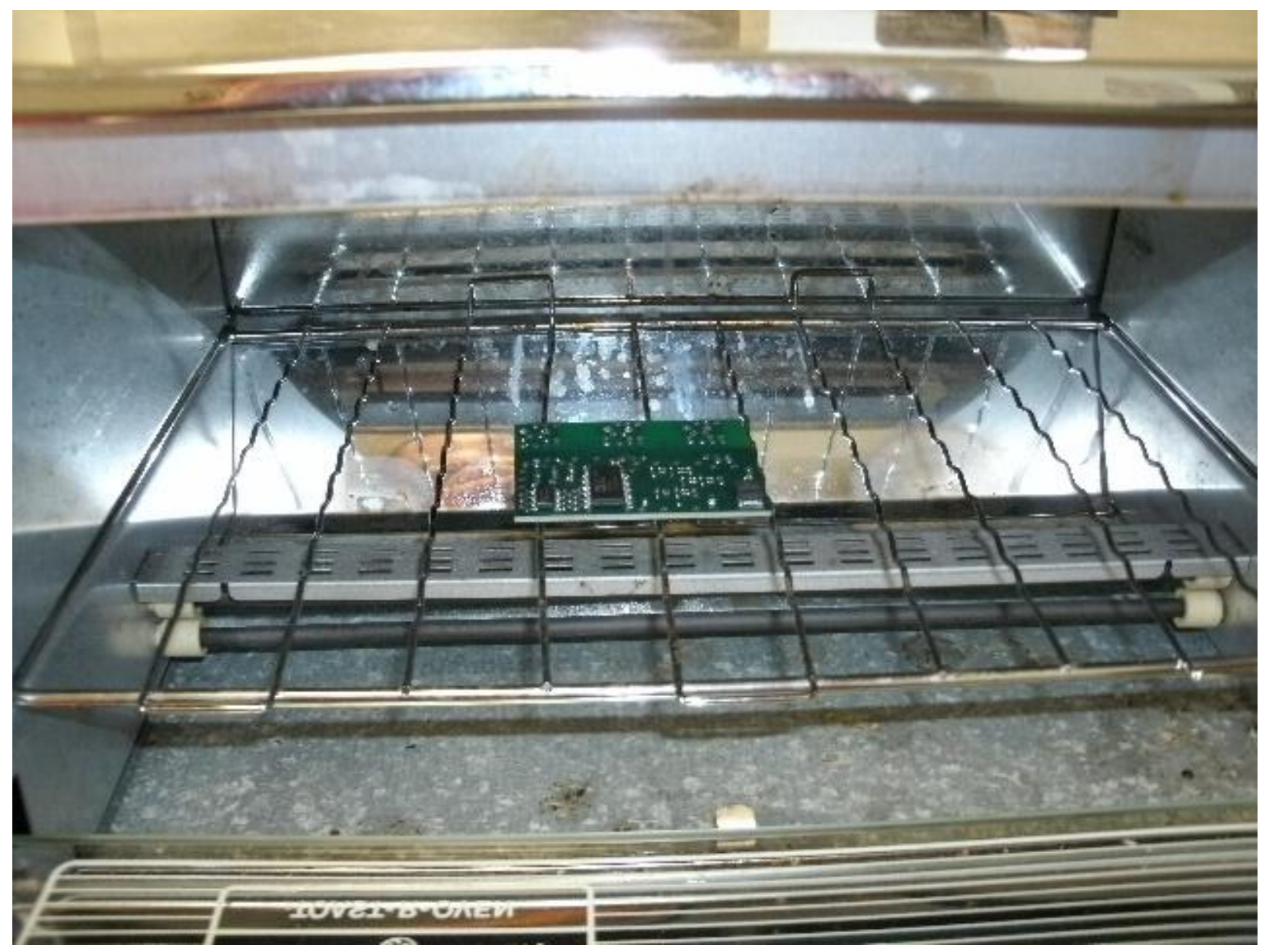

Figure 19: Board in oven with all of the surface mount parts in place ready for solder reflow 


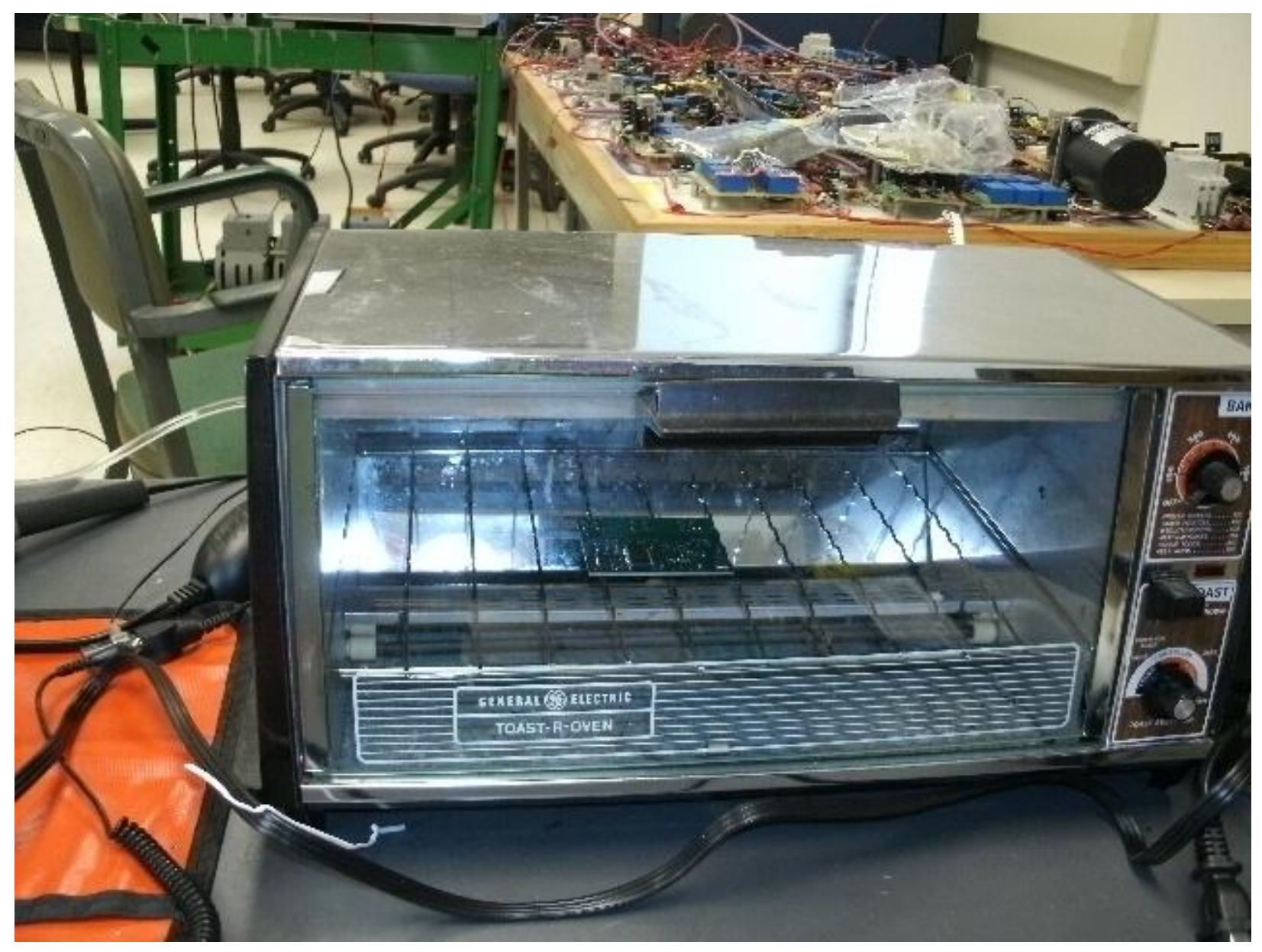

Figure 20: Placement of board in oven

After all of the surface mount parts have been place the board is put in the oven. In the toaster oven used the board was placed in the center of oven on the wire rack only then with the door closed the oven is set to toast on maximum while watching the board to see that the solder paste all melts. A flashlight is handy during this step. All of the solder will not necessarily melt at the same time, there is usually a progression from the small parts through to the largest ones, but it will depend a bit on where in the oven the board is placed, the center of the oven seems to work the best. As soon as all of the solder has melted as indicated by smooth shiny fillets at all of the junctions, the oven is shut off and the door opened to allow the part to cool. 


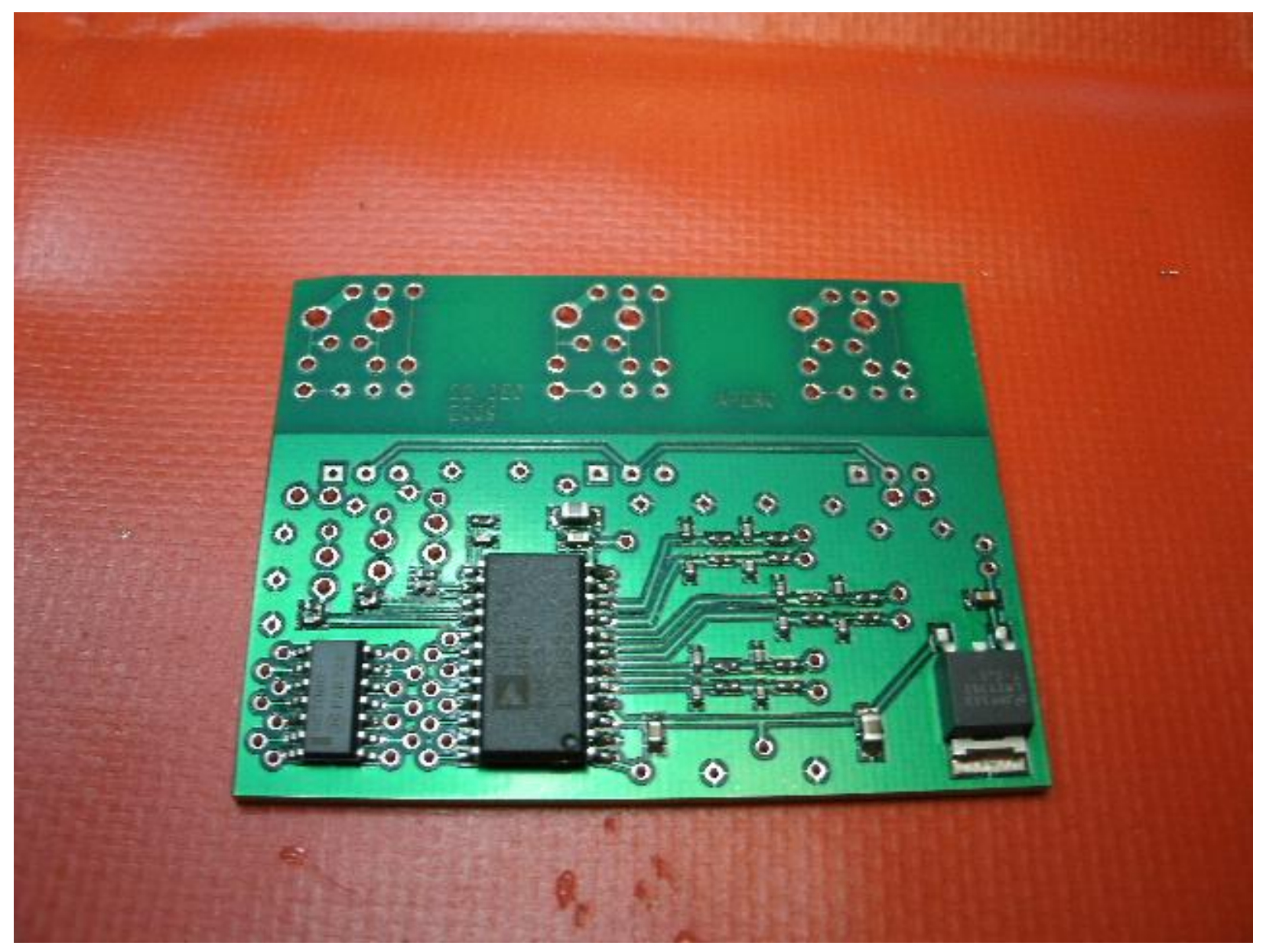

Figure 21: After cooling a satisfactory SMT soldered board, note the two resistor capacitor pairs that are angled together probably due to just a little too much solder on the right side of each

The part is then inspected for solder bridges or other defects. Occasionally there will be a solder bridge between adjacent pads, but those can be reworked as necessary. To minimize the bridges requires a little practice; less solder paste will result in fewer bridges, but may leave an unsoldered pin. As a rule, erring on the too little side is probably best since it takes less solder paste to make the actual bond than is usually assumed. Placement of the parts is also not as critical as it would appear at first because of the strong cohesive force of the molten solder, the parts will often move slightly to their correct position. There are times however on small two contact devices like the resistors and capacitors where there will not be enough solder paste or a good enough contact with the flux on one pad and the cohesive force will stand the part on end. This can be easily reworked too. 


\subsection{Reworking Surface Mount Parts}

With practice a high percentage of the boards produced do not need to be reworked and can proceed to the next step of assembly. Reworking the SMT (Surface Mount Technology) boards requires heating them to just below the solder liquefaction stage and then applying a little extra heat via hot air to melt the solder allowing the parts to be moved with a careful hand and a pair of tweezers.

The preheat device is a simple travel iron that the boards lay flat on with the SMT parts facing up and the heat traveling up through the bottom of the board. It's important that the boards lie flat to make good thermal contact with the hot surface of the iron so it's best to do the rework before any of the standard soldered parts are installed that would keep the board from making good contact. The hot air source is a modified RadioShack® desoldering tool with air supplied by an adjustable flow aquarium pump. The air is adjusted so there is enough to transfer heat but not so much as to blow the parts around, usually a little less is required for the smaller parts for this reason. The rework tools can also be used to solder individual parts rather than using the toaster oven, but is not practical when many parts need to be done at on a single board. Rework tools are shown in Figure 22 and Figure 23. 


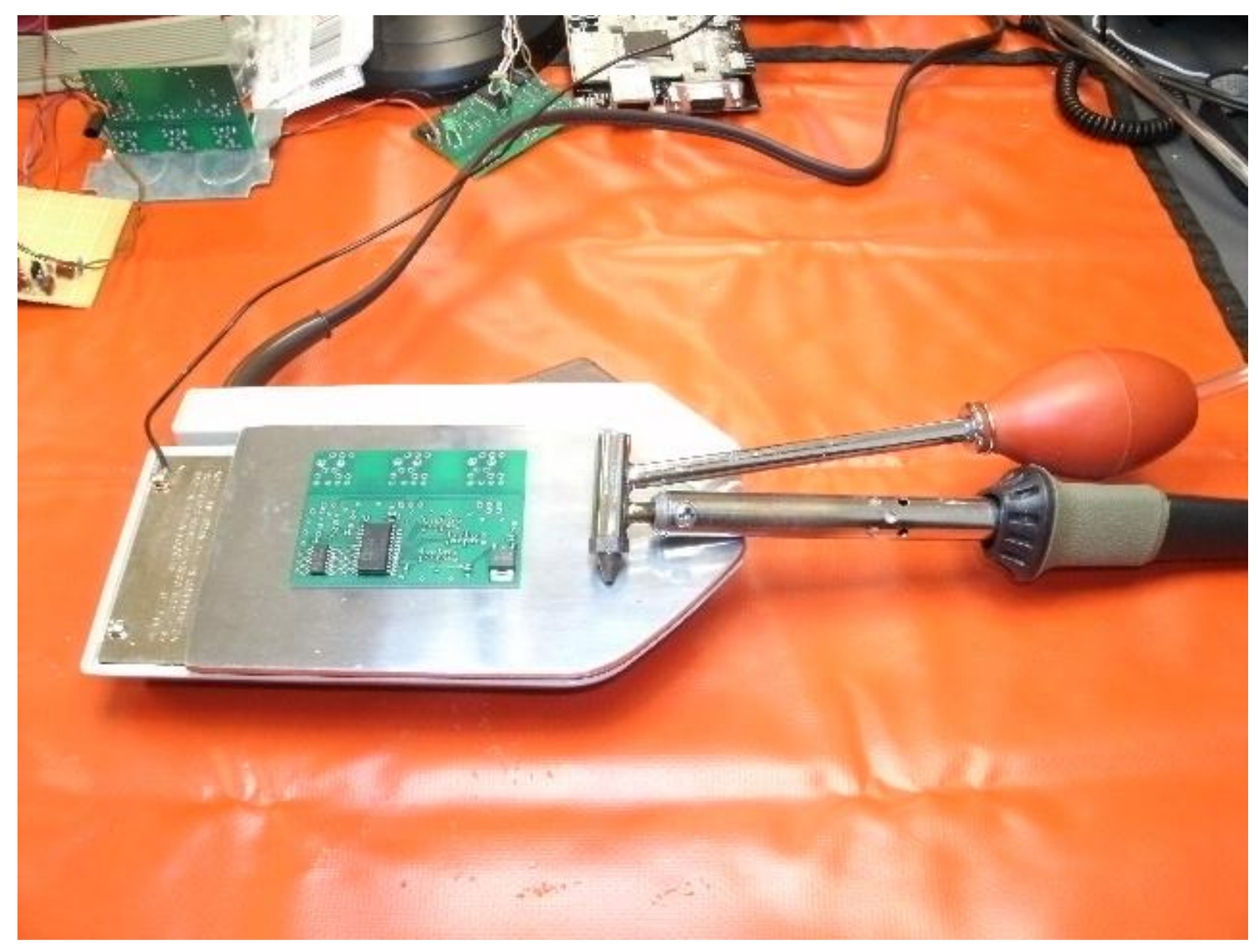

Figure 22: SMT printed circuit board rework setup 


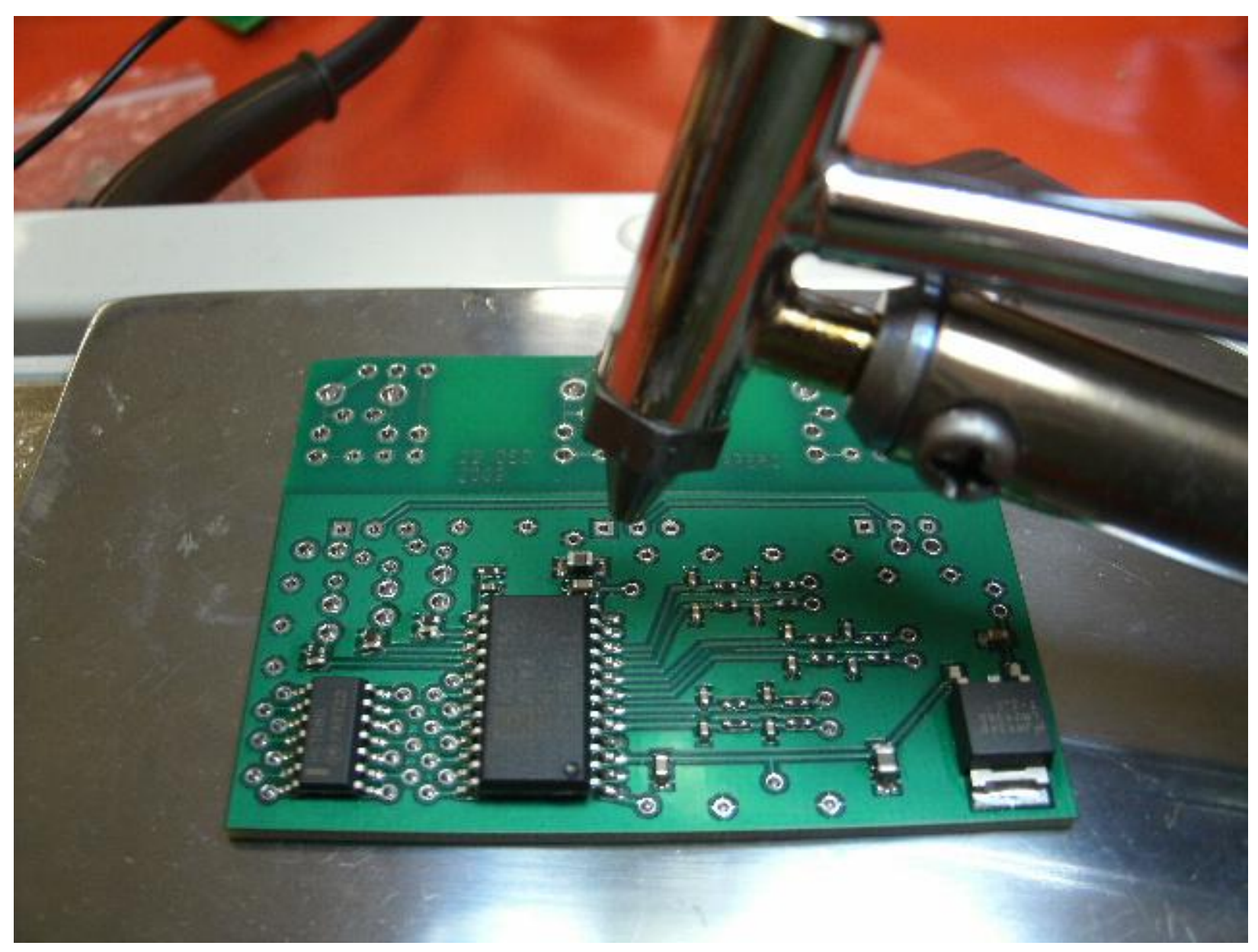

Figure 23: SMT printed circuit board rework setup closeup

Credit for the rework apparatus must be given to Will O'Brien at:

http://www.engadget.com/2006/03/07/how-to-make-a-surface-mount-soldering-iron/ and information from http://store.curiousinventor.com/guides/Surface_Mount_Soldering/Tools which mentions using the toaster oven for reflowing solder also mentioned at many other sites.

\subsection{Final Assembly}

If all of the parts are in the correct place with good solder contact and no bridges the board can go on to have the opto-isolators, triacs and other parts and wiring soldered using a conventional soldering iron

The boards were designed to fit in a standard electrical light switch box that serves as a heat sink for the triacs and provides strain relief for the main power cables and a mounting platform for the MCU demo units. 


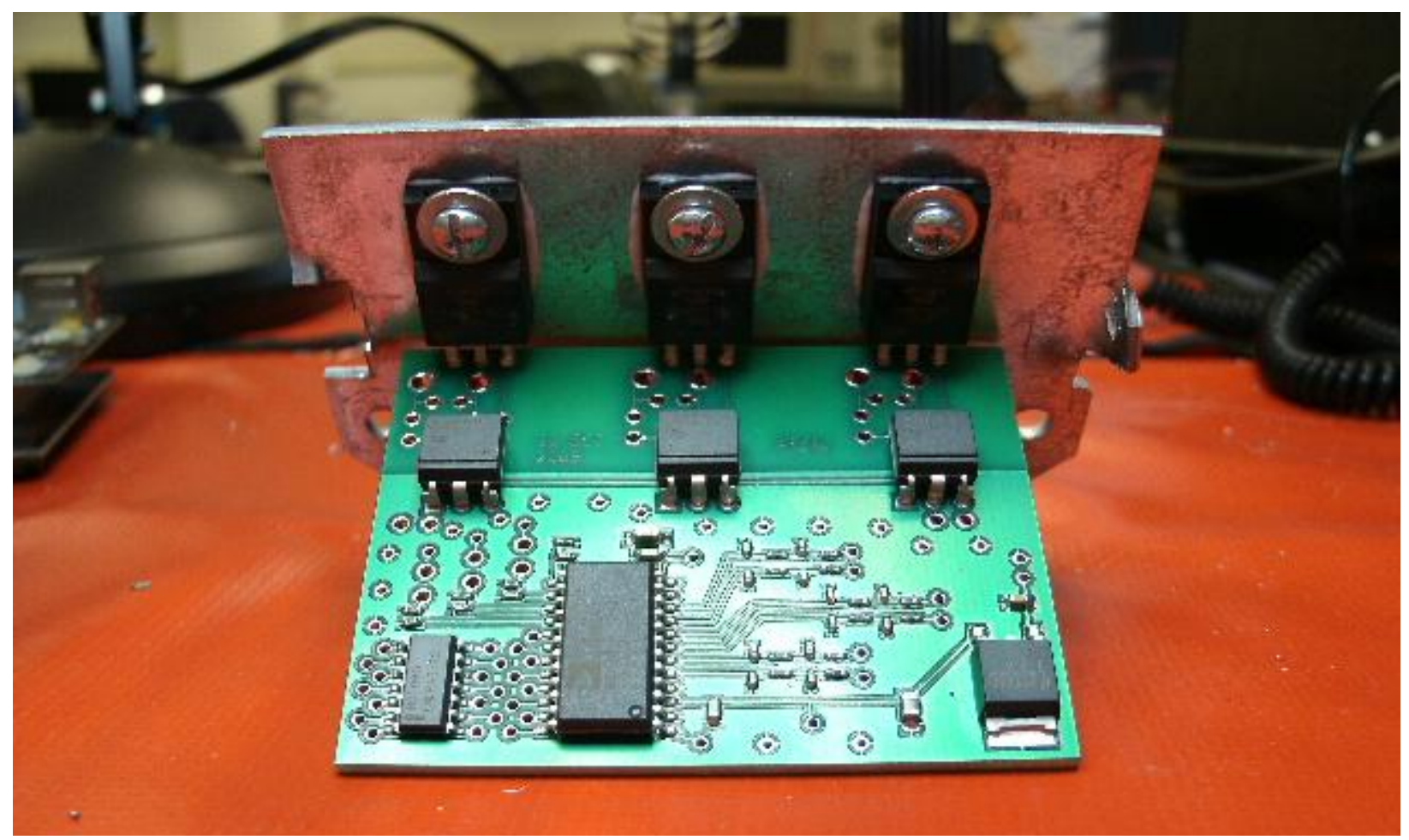

Figure 24: The sense and control board with SMT parts, opto-isolators and triacs installed on heat sink/enclosure side piece

The finished modules are small and light enough to attach directly to the switches that they parallel. They are powered by $\sim 5.5 \mathrm{VDC}$ which can run on an unused pair on the Ethernet cable going to each module. The wiring between the MCU module and the sense and control board is shown in Table 3: Wiring between the M52259DEMOKIT and the sense and control board

Table 3: Wiring between the M52259DEMOKIT and the sense and control board

\begin{tabular}{|c|c|c|}
\hline Signal Function & $\begin{array}{c}\text { Pin number on J4 } \\
\text { M52259DEMOCOM board }\end{array}$ & $\begin{array}{c}\text { Pin on 74LVC08AD sense and } \\
\text { control board }\end{array}$ \\
\hline +3.3VDC & 1 & 14 and collector of 2n2222 \\
\hline Ground & 3 & 7 and board ground \\
\hline Triac trigger & 14 & 9 and 10 \\
\hline IRQ & 18 & 6 \\
\hline DOUT(MCU) & 19 & ADE7758 Pin 22 \\
\hline DIN(MCU) & 23 & 3 \\
\hline CS & 24 & ADE7758 Pin 21 \\
\hline SCLK & 25 & ADE7758 Pin 23 \\
\hline
\end{tabular}




\subsection{Intelligent Switch Module Cost}

Table 4: Parts breakdown for one intelligent electronic switch are as follows

\begin{tabular}{|c|c|c|c|}
\hline Part & Number & Unit cost & Total cost \\
\hline M52259DEMOKIT & 1 & $\$ 49.00$ & $\$ 49.00$ \\
\hline PCB & 1 & $\$ 7.40$ & $\$ 7.40$ \\
\hline ADE7758 & 1 & $\$ 8.95$ & $\$ 8.95$ \\
\hline Switch Box & 1 & $\$ 2.86$ & $\$ 2.86$ \\
\hline 5V Regulator & 3 & $\$ 1.30$ & $\$ 1.30$ \\
\hline Triac & 3 & $\$ 0.64$ & $\$ 1.92$ \\
\hline Opto-isolator & 1 & $\$ 0.84$ & $\$ 2.52$ \\
\hline Level shifter & 2 & $\$ 0.48$ & $\$ 1.58$ \\
\hline 10 uF Cap. & & $\$ 0.79$ & $\$ 1.00$ \\
\hline Resistors estimate & & & Total $\$ 78.01$ \\
\hline Hardware estimate & & & \\
\hline
\end{tabular}

\subsection{Current Sensor Design}

In an effort to keep cost low and size small current sensors were hand wound on salvaged toroid cores for initial testing. The integration method of current measurement was chosen to keep part count down and power dissipation at a minimum since that power would have to be dissipated in the electronic switch. The sensors are simple transformers wound on the toroidial core with about 85 turns of $30 \mathrm{AWG}$ as the secondary which gets attached to the ADE7758 A/D and about 3 turns of the 14 AWG that connects to the triac. This arrangement makes a very compact transformer that fits easily in the switch box and is very tolerant to noise pickup because of the toroidial configuration. The current sensors being tested are shown in Figure 25. 


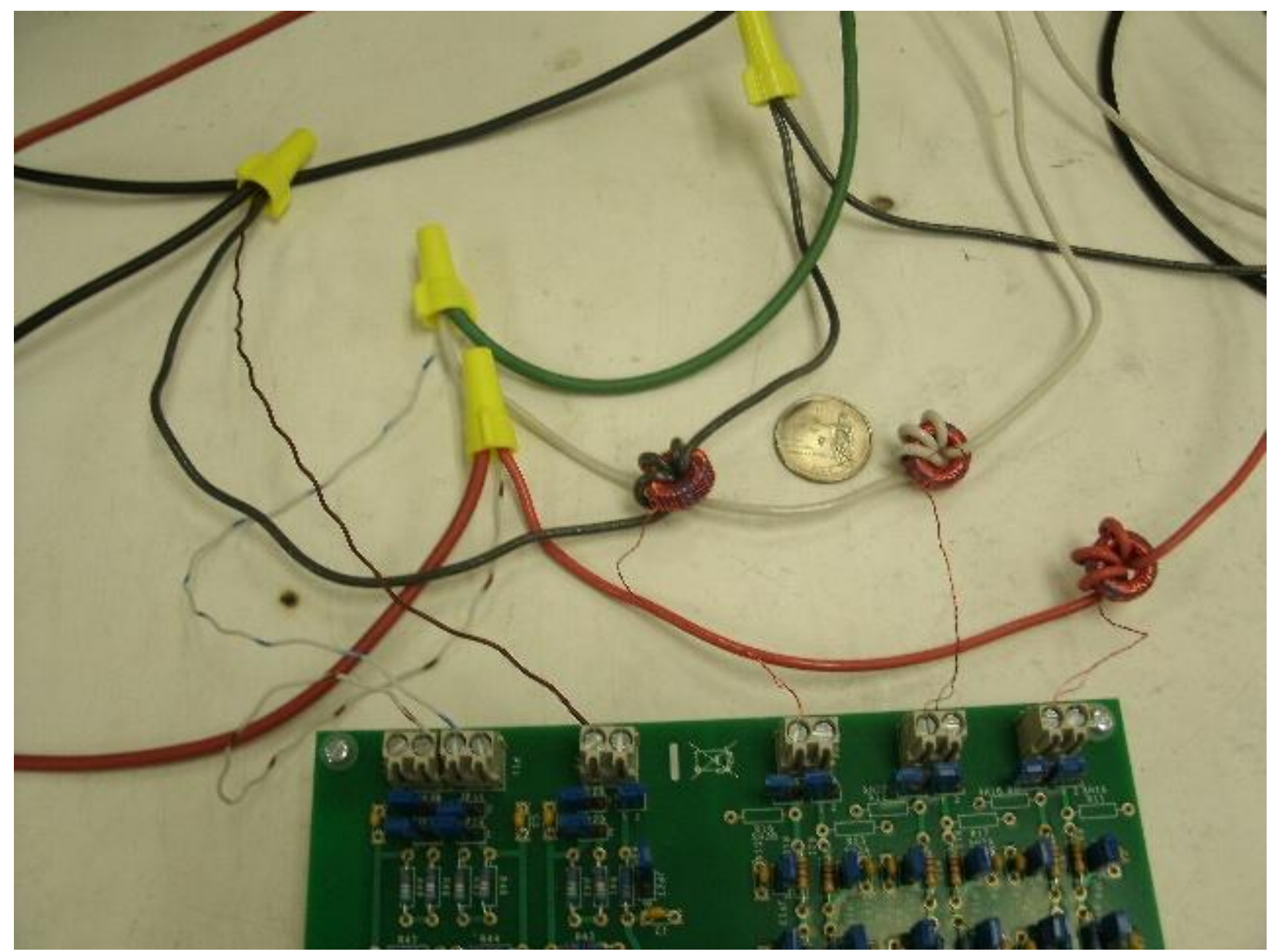

Figure 25: Current sensors being bench tested with the ADE7758 evaluation board

\subsection{Clock and Distribution Circuit Design}

In addition to the DC power needed by both the MCU and the meter chip, each meter chip needs a clock signal of about 10MHz; optionally each MCU can be driven by an external clock as well. It was decided that it would be of great benefit to at a minimum synchronize all of the metering chips so a $10 \mathrm{MHz}$ clock will be distributed on the other unused pair of the Ethernet cable. The Ethernet cable has an impedance of $100 \mathrm{ohms}$ which requires a carefully designed driver to supply the clock signal at the roughly 3 volt swing required by the meter chip. An off the shelf IC was not found that would work in this situation so a discrete transistor buffer and amplifier is being designed to perform this function. 


\section{Chapter 7 Hardware Test and Results \\ 7.1 Sensor Testing}

The voltage sensors for this system are simple voltage dividers and the A/D's have very high input impedance so they have no appreciable effect on the voltage sensed.

The current sensor configuration chosen was novel so a bit of experimentation was required in their design. An evaluation board for the ADE7758 was purchased from Analog Devices. It has already proven very helpful in the development of the intelligent switches. The board comes with LabVIEW based software with a GUI that makes it very easy to access all of the chip's capabilities. Analog Devices was generous enough to email a copy of the source code which has the potential to be extremely useful. The only downside to the evaluation board is that it requires a parallel printer port for an interface to the board which is rather rare anymore. It is hoped that the source code can be modified to use an Ethernet port instead in which case the ADE7758's could be accessed though the MCU's in the intelligent switches.

Since there was no data on the material of the toroid cores used, no real analysis was employed in their design. However after only a couple of iterations a windings ratio was settled on that produces a faithful representation of the current in the range that will be found on the simulator at a voltage that matches the A/D input very well. The evaluation board setup to test sensors is shown in Figure 26. 


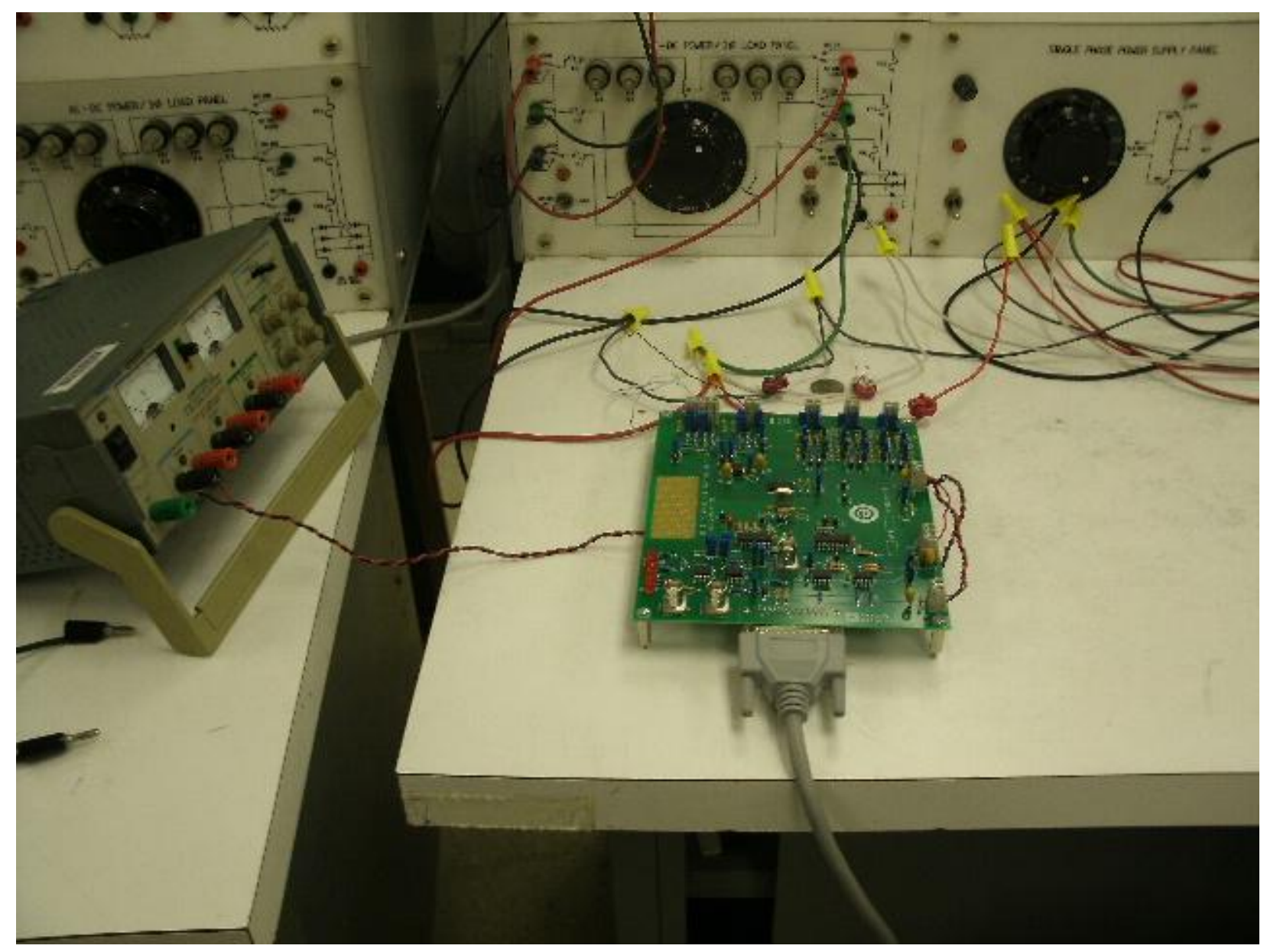

Figure 26: Sensor test setup using ADE7758 evaluation board

To explore the linearity of the ADE7758 and the current and voltage sensors the evaluation board was set up on one of the laboratory benches. Three phase power can be easily routed through variacs to change the voltage and switchable resistive, inductive and capacitive loads. The three phases sampled by the evaluation board were also sampled by a Yokogawa PZ4000 power analyzer.

The LabVIEW software only displays numbers from the A/D's and the end user is expected to know how to scale them to display the voltage and amperage they represent. The ADE7758 does have gain and offset compensation so that phase to phase amplitude is accurate once these parameters are set during calibration.

2000 samples were taken by the ADE7758 and 2 seconds worth of data sampled by the PZ4000. Only RMS values were looked at for both the current and voltage because the 
LabVIEW GUI gave more precise values in that mode since it is an accumulated result. The voltage was changed incrementally from 0 to 150VAC and the current and voltage and the corresponding counts from the ADE7758 were recorded. The data was rearranged in Microsoft Excel and plotted.

Figure 27 shows the counts versus voltage over the range.

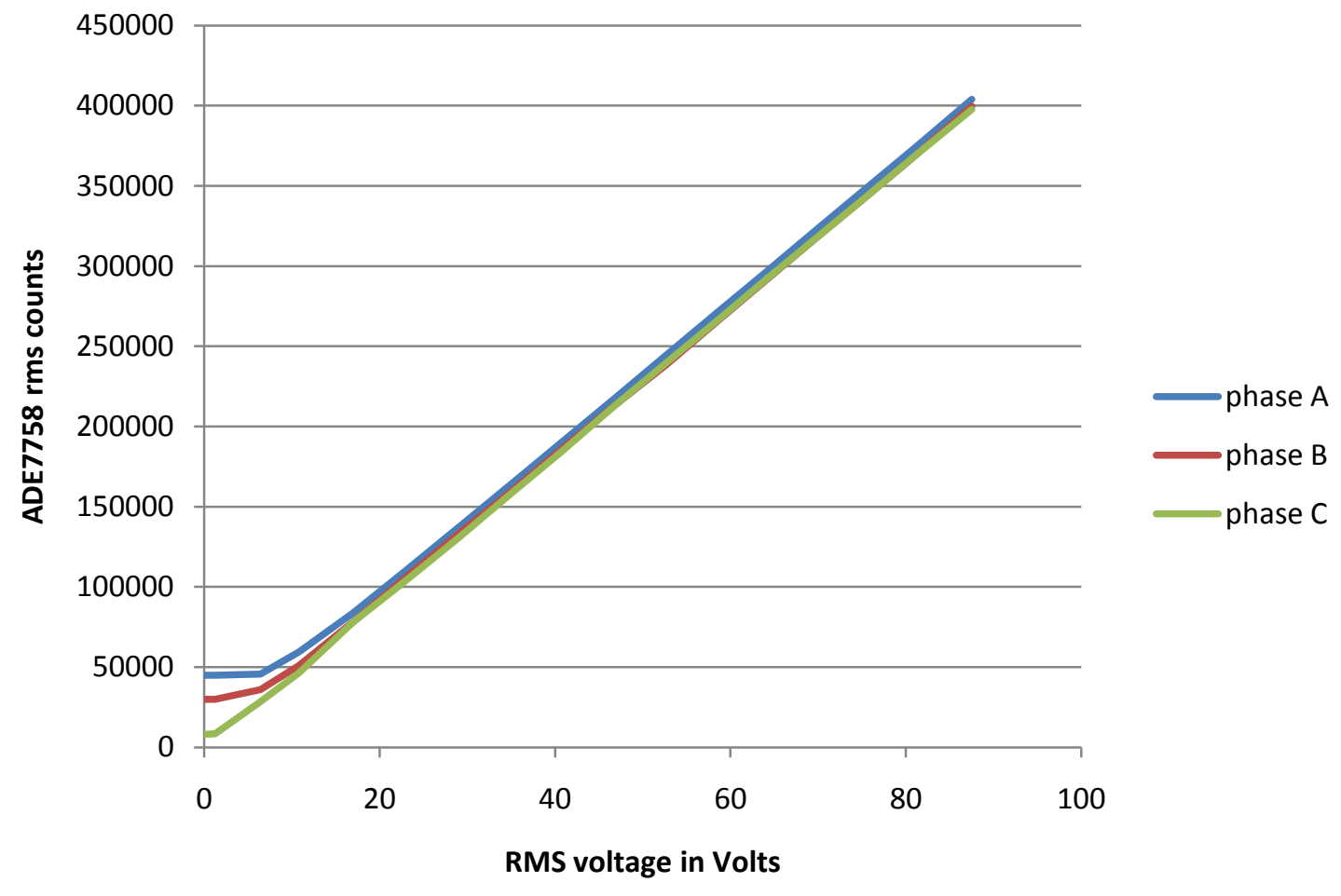

Figure 27: ADE7758 counts versus voltage

The plot is very linear as can be seen until a noise floor is reached at the low end of the range below 10VAC. It is interesting to note that phase A has a considerably higher noise floor. 
Figure 28 is a plot of the counts per volt versus voltage. The data below 10 volts was eliminated to focus on the more linear range of the setup.

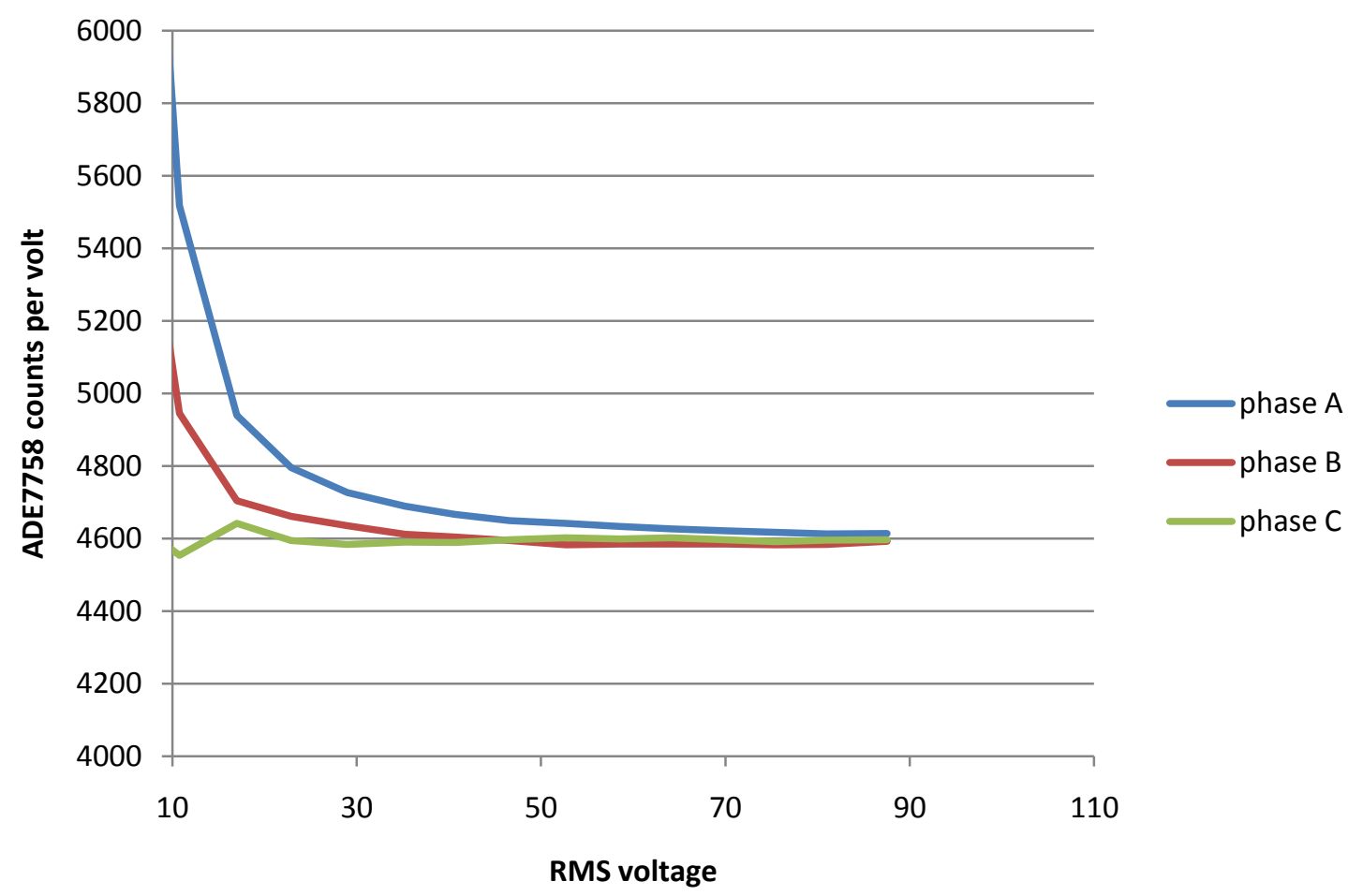

Figure 28: ADE7758 counts per volt vs voltage 
Figure 29 shows the counts versus current of the ADE7758 test setup with the hand wound transformers in the integrate configuration. The device appears to be very linear from this view. The interesting thing to note is the difference in slope between the three phases. Since the gain on all three channels was the same it can be assumed that the difference is due to a difference in transformers.

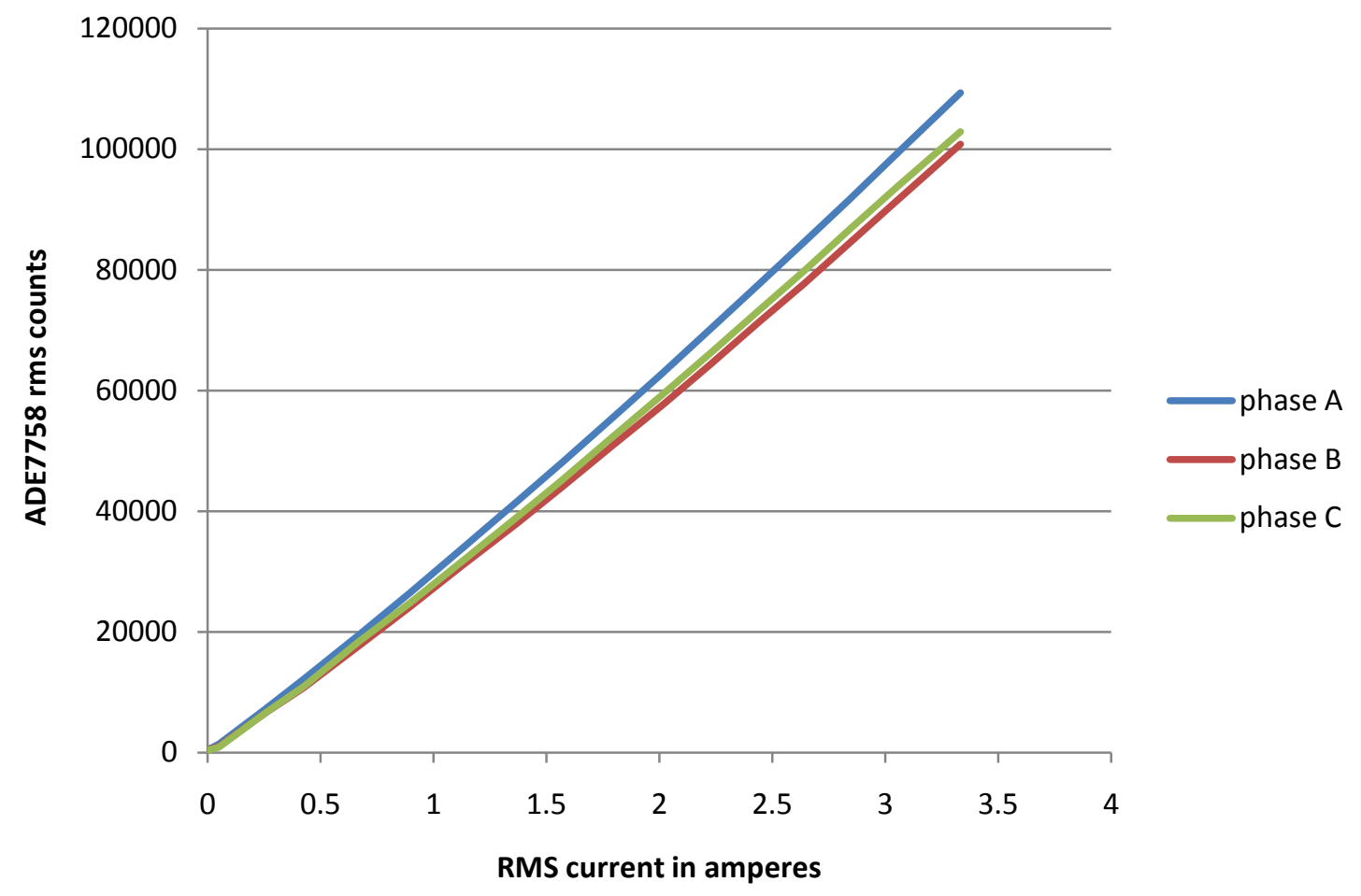

Figure 29: ADE7758 counts versus current

It is very possible that they did not all get the same number of turns. This can probably be fixed with individual channel gain adjustments or it may be found that more care is needed in winding of the transformers. This setup may be useful in the long run as a final test that the transformers have the correct number of turns. 
Figure 30 shows the counts per amp sampled by the ADE7758. With this plot it's easy to see the relative difference in gain between the phases, as well as a general curve across the amperage range tested. This may be due to some nonlinearity in the core material.

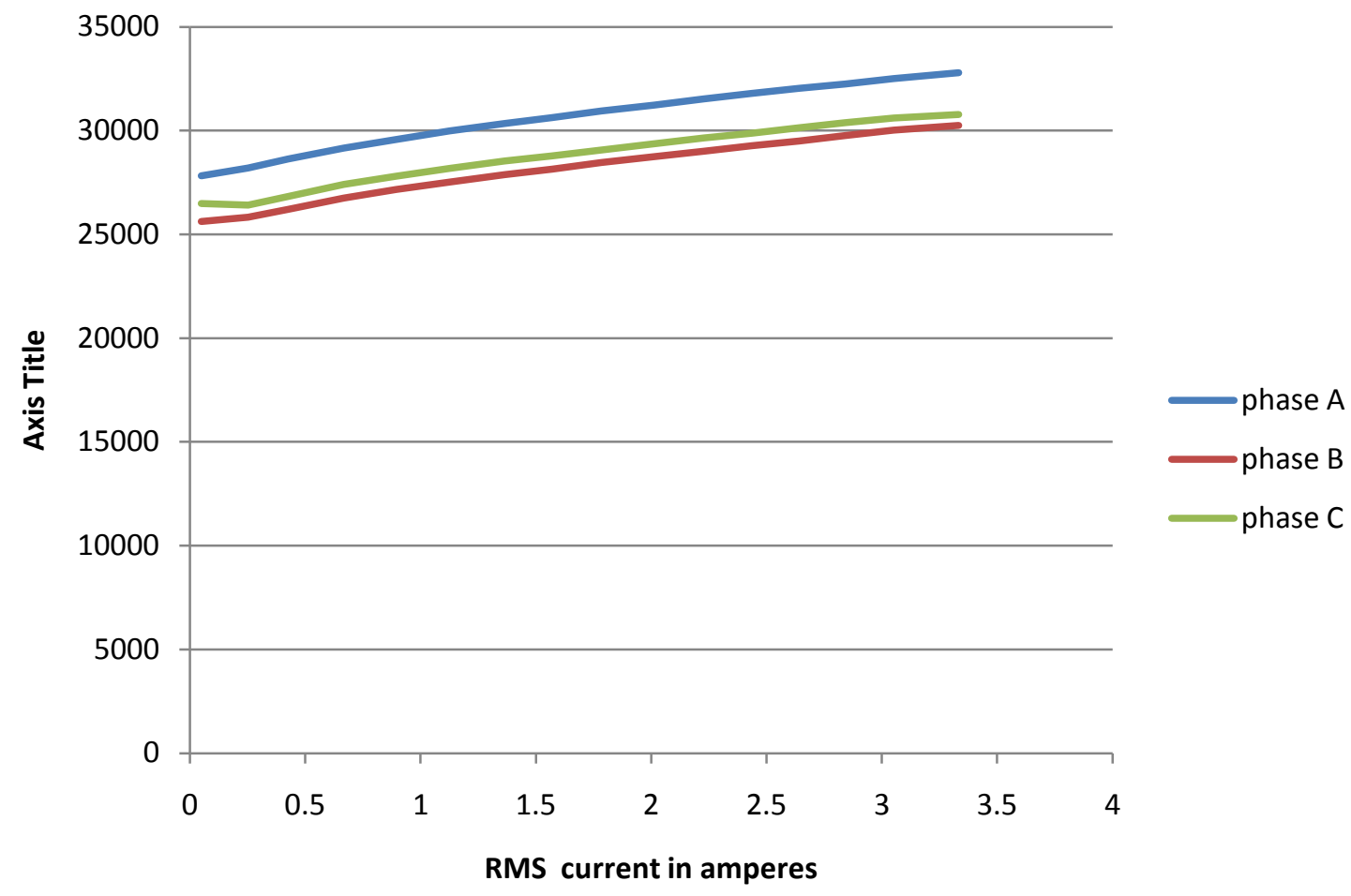

Figure 30: ADE 7758 counts per amp vs amperage

It probably should be noted that on both the current and voltage side the counts directly from the $\mathrm{A} / \mathrm{D}$ are a small percentage of the maximum available. The peak to peak values that correspond to the maximum current of this setup which is just under $3.5 \mathrm{amps}$ is only $-181,000$ to $+181,000$ which is nowhere near the $-2,642,412$ to $+2,642,412$ capable from the 24 bit A/D. The voltage counts for this setup are even less, -2320 to +2700 . More careful selection of input voltage dividers may make the noise floor seen here insignificant.

\subsection{Communications Testing}

While the major open issue is the programming of the MCU to ADE7758 SPI interface there has been some success modifying example code from Freescale that allows access to the 
serial ports of the M52259DEMOKIT through their Ethernet port. By assigning individual IP addresses to several of the demo boards communication though their RS232 serial ports was possible. This alone may allow use of the demo kits with the digital relays that are only equipped with RS232 ports to give them added communications ability and the same intelligence as the new switches. The setup is shown in Figure 31. The demo kits can all be connected through a hub or router and addressed individually through their Ethernet port by their unique IP address. The serial connection is made to any machine that has a RS232 com port. The program simply repeats text typed in one format into the window of the other. The lab that this is taken from is lab 6 Ethernet to Serial Bridge, Freescale MQX RTCS in the MQX tutorial (9).

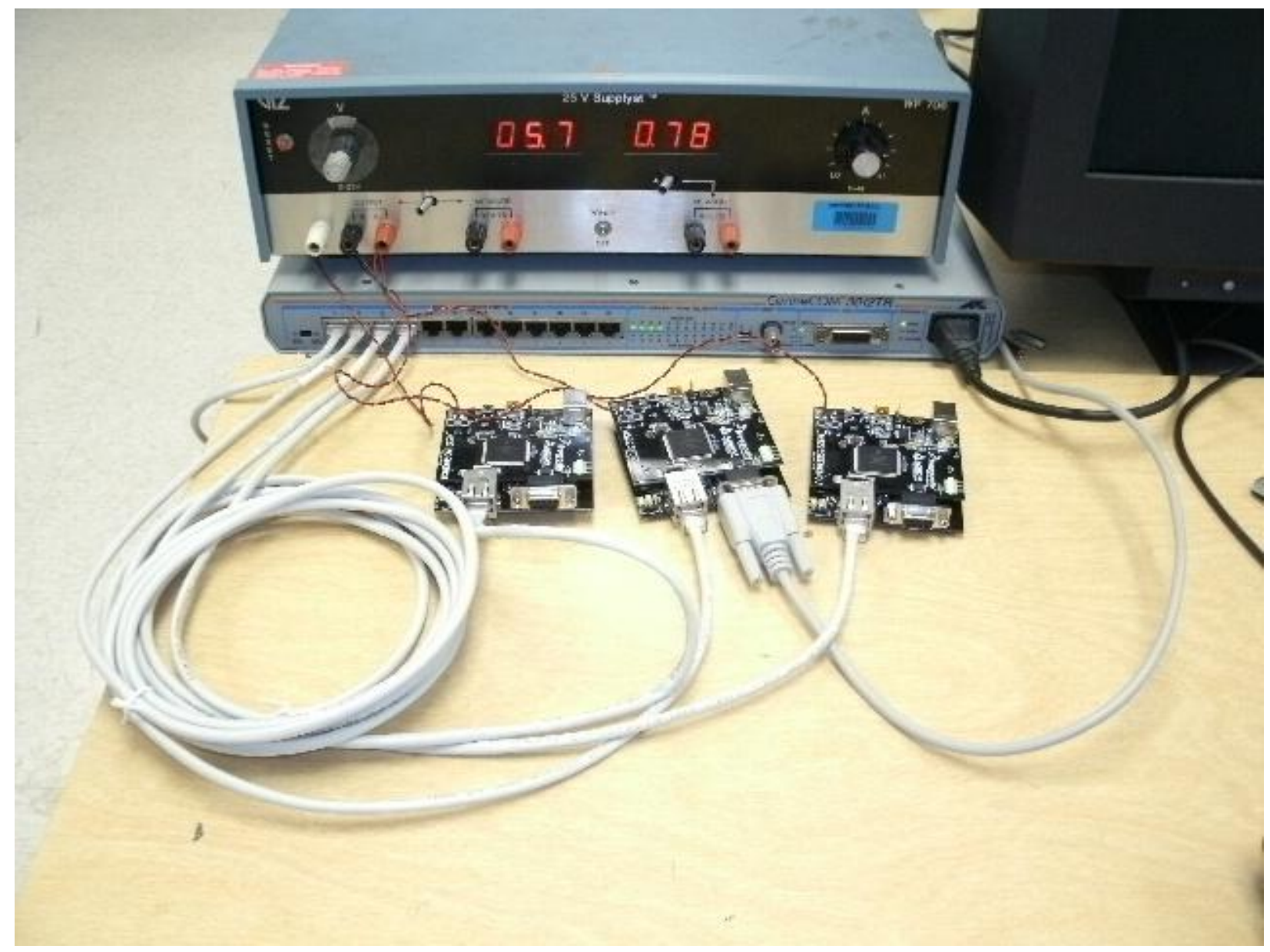

Figure 31: Test setup for Ethernet to serial communications rerouting

\subsection{Triac Switch Performance}


To test the triacs a sample code that turns an LED on for one second then off for the next was used to do the same with the triacs on the simulator. The entire load that was available on the simulator was switched in gradually and the triacs performed as hoped even. The circuit was modified for $120 \mathrm{VAC}$ by changing the gate resistors to $1.8 \mathrm{~K} \mathrm{ohm}$ instead of the $10 \mathrm{~K}$ that were in place for the 750VAC of the simulator. The setup was then moved to one of the student benches where three phase at 120VAC was switched in the same way and the turn on and turn off of current and voltage waveforms was captured with a Yokogawa PZ4000 power analyzer.

The turn on waveforms were just as expected with the addition of some noise that wasn't considered but can be easily explained see Figure 32. The turn on is immediate as expected but the crossovers and distortion in the other phases at the same time are not would be expected from a normal three phase power sinusoid waveform at first glance. The delay in turn on after each cycle goes through zero is due to the finite current require though the gate to trigger the triac, until the voltage has reached a point where that current is attained there is no conduction. The reason that the other phases are distorted during that time is that this is a three phase delta wired circuit soothe sum of the currents and voltage must be zero at all times, if there is no current flow in one phase, effectively an open the other phases will have to sum to zero regardless.

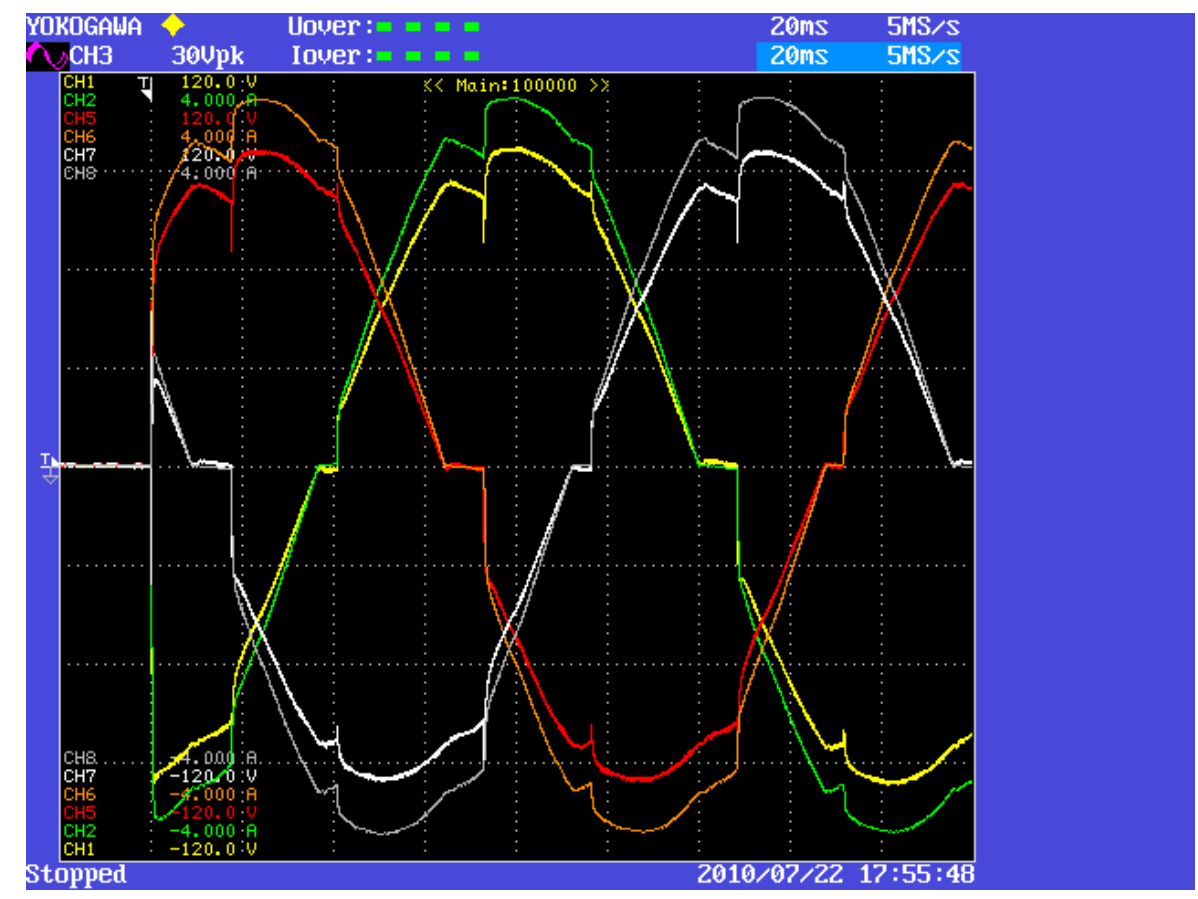

Figure 32: Three phase triac turn on waveforms with resistive load 
The turn off wave form although also not exactly what was expected make sense in light of the previous argument, see Figure 33. The triacs lose their trigger at the mid way point of the plot and the first triac turns off, channels 5 and 6 as expected. Immediately after channels 1 and 2 change their curvature to counter that of channels 7 and 8 in order for the sum of the voltages and current to equal zero.

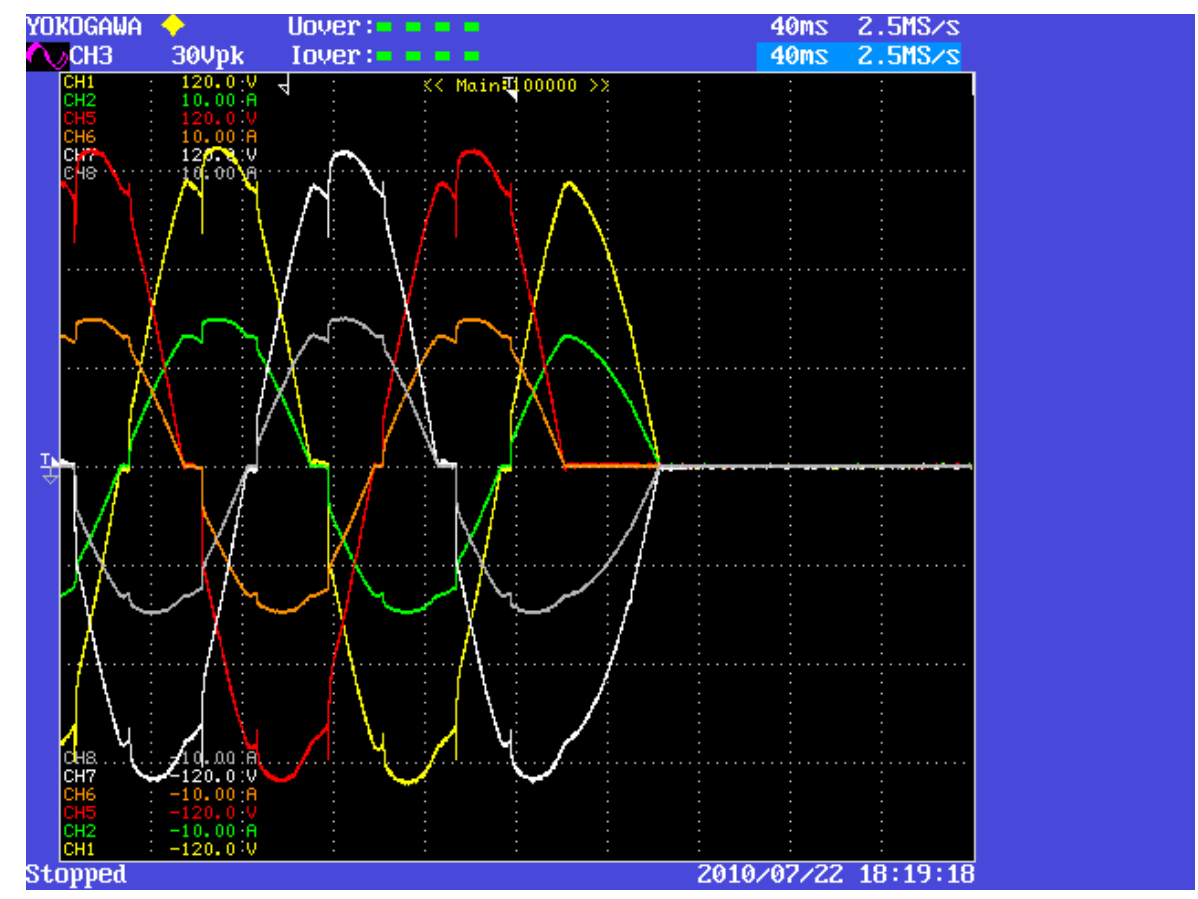

Figure 33: Three phase triac turn off waveforms with resistive load

The turn off with an inductive load is as is widely described in the literature, see Figure 34. In this case the trigger happens at the first division, or $10 \%$. The current in each phase shuts off as expected, but the voltage in each phase tapers off very slowly. This is due to the voltage induced across the inductor and resistor in parallel as the magnetic field collapses in the inductor. 


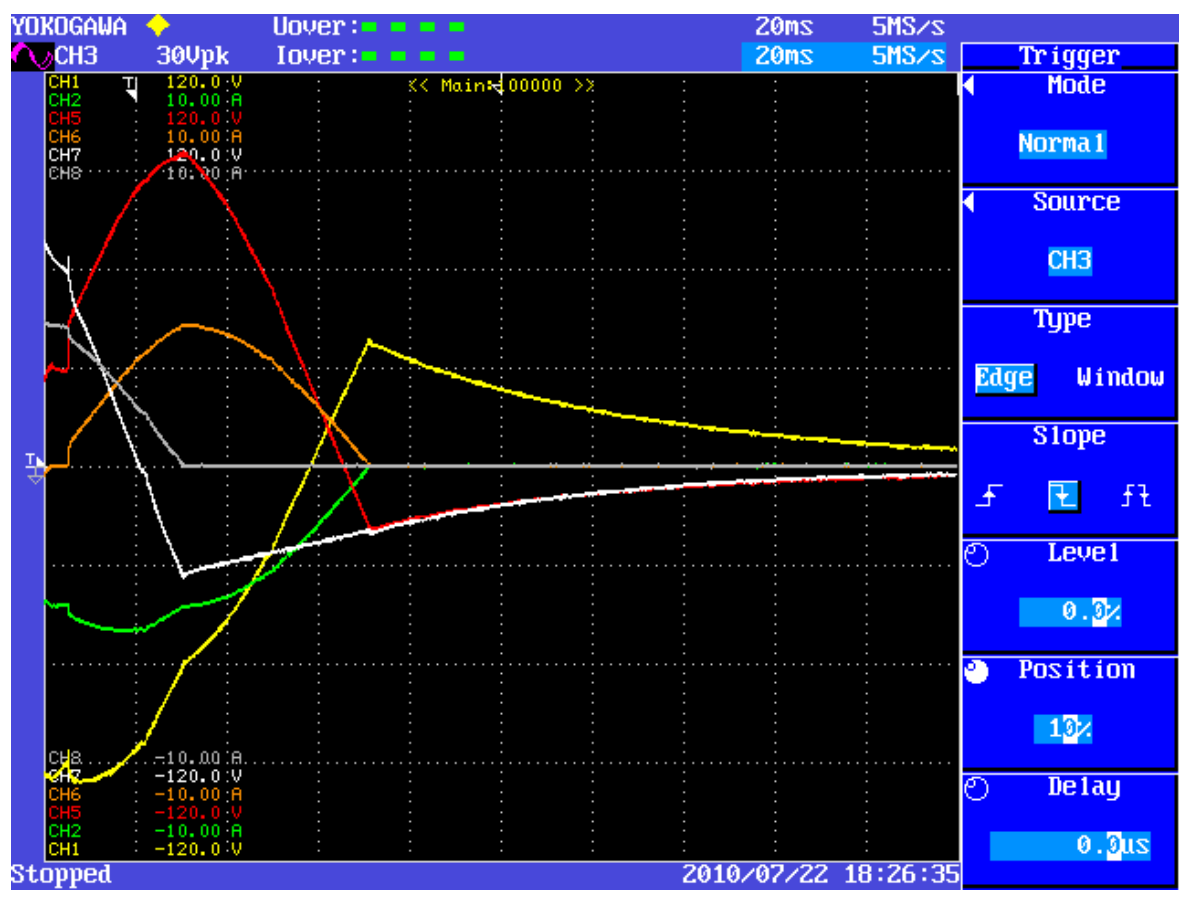

Figure 34: Three phase triac turn off with inductive load

\subsection{SPI MCU to ADE7758 Interface}

The ADE\&\&58 metering chip talks to the MCU through a QSPI queued serial peripheral interface. The MCF5229 MCU comes with that as one of its many communications ports, but the actual implementation of the protocol is not necessarily straight forward like plugging in a monitor to your computer, so a test setup was assembled to be able to work just with that particular interface and not have to worry about harming any other devices in the process. The setup is shown in Figure 35. The interface is fixed as far as the ADE7758 is concerned but there are parameters that need to be set on the MCU side via Freescale's CodeWarrior Development Studio software. This is currently being worked on and is expected to be the last hurdle to having a working prototype. 


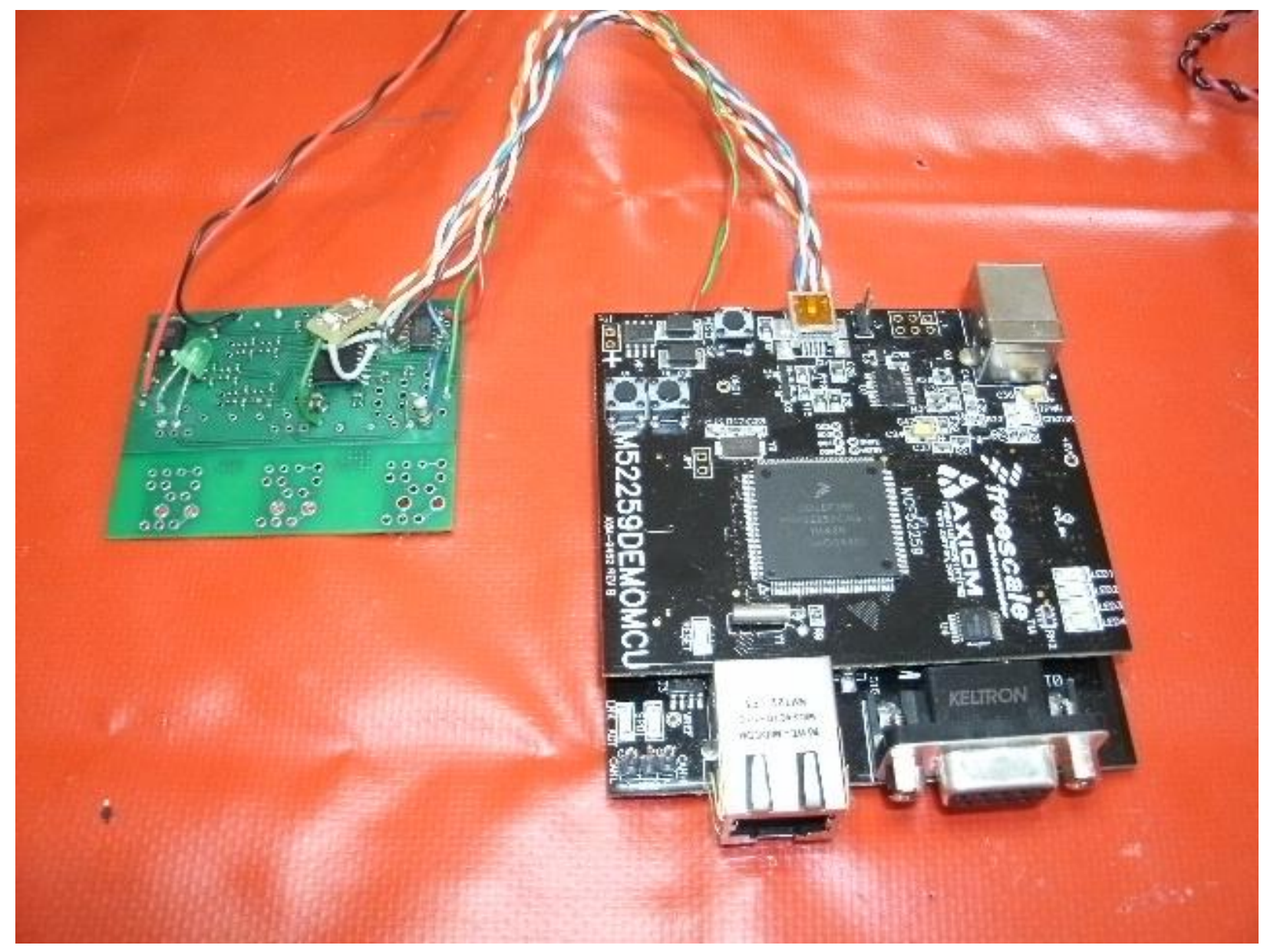

Figure 35: SPI communication interface test setup 


\section{Chapter 8 Conclusions}

\subsection{Successes}

\subsubsection{Digital Relays in Place}

The digital relays are in place and ready to be wired up and used in labs or connected in with the intelligent switches for algorithm testing. The wiring should not be a difficult task, but will require some attention to detail and be documented on the schematics for the simulator.

\subsubsection{Sense and Control Board}

The sense and control boards have been designed and fabricated. A procedure is now in place for the assembly of the boards. All testing done on them has been successful. The open issues are the SPI interface and the verification of the sensor filter circuits which require some sort of communication with the ADE7758 on the board so may be best left until that issue is resolved. The 50 boards that are in house are all suitable for use. If a next version of the board is made and incorporates the new layout of the level shifter and transistor buffer it would probably save some time and make for a neater package.

\subsubsection{Designed, Fabricated and Tested Current Sensors}

The current sensors seem to work just fine, they give a voltage that's in a good range for the ADE7758 A/D's and have been shown to be acceptably linear over the range of currents expected. There may be however room here for improvement with a more attention paid to core selection and size. More experience with the present devices may indicate that more or less coupling would be advantageous on the power simulator. I may also be found that more than one type would give the greatest benefit. The evaluation board will be a great tool for exploring this topic in more detail

\subsubsection{Triggered Triacs with MCU under Full Load}

The triacs performed very well and despite the added noise induced at crossover look to be a very satisfactory solution as AC switches on the simulator. There is also room for experimentation and possible improvement through the design and testing of snubbers for switching more inductive loads if needed in the future, and the impact of zero crossing optoisolators would also be an interesting topic to explore further. 


\subsubsection{Modified Code for Serial to Ethernet Operation}

This unfortunately so far is about the only success as far as software goes it is however a big step towards communicating with the digital relays both through and with the MCU's.

\subsection{Open Issues}

\subsubsection{ADE7758 to MCU SPI Interface}

The SPI interface between the MCU's and the ADE7758's is the fundamental hurdle that stands in the way of starting to write code to test reconfiguration algorithms and will be the next issue addressed. The next step will be to contact Freescale to ask for advice from their engineers on getting a handle on programming the MCU's. It may actually be something pretty straight forward after some hints on approaching the issue from someone who is more familiar with the devices.

\subsubsection{MHz Clock Distribution}

This is really an optional endeavor, but in the long run something I think is really worth implementing now. A clock could be taken from the MCU board and testing could proceed until phasor information was desired, but it is much easier to do now, not much more difficult than wiring a clock from the MCU, so the effort may as well be put into the synchronized clock approach. I have some ideas about using the LC model of the CAT 5 transmission line in the circuit that would be interesting to explore, and may be useful at much higher frequencies and smaller scale clock distribution.

\subsection{Future Work}

From the beginning I have thought of this project as a chalk board where an endless number of ideas could be explored. Every decision that was made was made with the intention of giving as many options and as much capability as possible. In that light I see no end to the future work that could be done with this hardware, I hope others do. 


\section{References}

1. Pant, Pradeep. Design and Real-Time Control of Shipboard Power System Testbed. Morgantown, WV : West Virginia University Libraries, 2007.

2. PAT: A Power Analysis Toolkit for MATLAB/Simulink. K. Schoder, A. Hasanović, and A. Feliachi. 1, s.l. : IEEE, Feb. 2003, IEEE Transactions on Power Systems, Vol. 18, pp. 42-47. 3. An Integrated Electric Shipboard Power System Testbed. P. Pant, K. Schoder, A. Feliachi. Arlington, VA, USA : s.n., 2007. Proceedings of the Electric Ship Technologies Symposium (ESTS). May 21-23.

4. Graeber, Brian. Web-Based Relay Management with Biometric Authentication. Morgantown, WV : West Virginia University Libraries, 2008.

5. Nareshkumar, Koushaly. Application of multi-agents to power distribution systems. Morgantown, WV : West Virginia University Libraries, 2008.

6. Multi-Agent Design for Power Distribution System Reconfiguration Based on the Artificial Immune System Algorithm. Rabie Belkacemi, Ali Feliachi. 2010. ISCAS 2010. May 30-June 2. 7. A Reconfigurable Distributed Multiagent System Optimized for Scalability. Summiya Moheuddin, Afzel Noore, and Muhammad Choudhry. 1, 2009, International Journal of Computational Intelligence, Vol. 5, pp. 60-71.

8. Analog Devices. ADE7758 Poly Phase Multifunction Energy Metering IC Data Sheet. s.1. : Analog Devices, 2008.

9. Freescale Semiconductor. M52259DEMOKIT: Low-Cost Demonstration Board Kit for MCF5225x documentation page. [Online] [Cited: July 28, 2010.] http://www.freescale.com/webapp/sps/site/prod_summary.jsp?code=M52259DEMOKIT\&fpsp= $1 \&$ tab=Documentation_Tab. 